\title{
Beyond gauge theory: positivity and causal localization in the presence of vector mesons
}

\author{
Bert Schroer ${ }^{1,2, a}$ \\ ${ }^{1}$ Present address: CBPF, Rua Dr. Xavier Sigaud 150, Rio de Janeiro 22290-180, Brazil \\ ${ }^{2}$ Institut für Theoretische Physik, FU-Berlin, Arnimallee 14, Berlin 14195, Germany
}

Received: 5 January 2016 / Accepted: 8 June 2016 / Published online: 6 July 2016

(C) The Author(s) 2016. This article is published with open access at Springerlink.com

\begin{abstract}
The Hilbert space formulation of interacting $s=$ 1 vector-potentials stands is an interesting contrast with the point-local Krein space setting of gauge theory. Already in the absence of interactions the Wilson loop in a Hilbert space setting has a topological property which is missing in the gauge-theoretic description (Haag duality, Aharonov-Bohm effect); the conceptual differences increase in the presence of interactions. The Hilbert space positivity weakens the causal localization properties of interacting fields, which results in the replacement of the gauge-variant point-local matter fields in Krein space by string-local physical fields in Hilbert space. The gauge invariance of the perturbative S-matrix corresponds to its independence of the space-like string direction of its interpolating fields. In contrast to gauge theory, whose direct physical range is limited to a gauge-invariant perturbative S-matrix and local observables, its Hilbert space string-local counterpart is a full-fledged quantum field theory (QFT). The new setting reveals that the Lie structure of self-coupled vector mesons results from perturbative implementation of the causal localization principles of QFT.
\end{abstract}

\section{Introductory remarks on origin and scope of string localization}

It is well known that the use of point-local massless vector potentials is incompatible with the positivity of Hilbert space. One usually resolves this problem by abandoning the positivity requirement of quantum field theory (QFT) while maintaining the point-local field formalism which leads to gauge theory (GT). The price to pay is well known from quantum electrodynamics (QED) in the standard indefinite metric (Gupta-Bleuler) gauge setting: positivity can be recovered

Dedicated to the memory of Raymond Stora and John Roberts.

a e-mail: schroer@zedat.fu-berlin.de for local observables, whereas charge-carrying fields remain outside its physical range. The separation between physical and unphysical quantum fields is done in terms of gauge symmetry, which is not a physical symmetry but a formal device to extract a physical subtheory.

Although the standard gauge formalism is restricted to the presence of vector potentials, the clash between zero mass point-local fields and positivity is a general phenomenon for all $s \geq 1$ zero mass tensor potentials. It does not affect the corresponding field strengths, but the short-distance dimension of the latter $\left(d_{s d}=2\right.$ for $\left.s=1\right)$ prevents their direct use in renormalizable interactions.

The alternative option is to accept the weaker localization required by positivity. The tightest covariant localization beyond point-local consistent with positivity turns out to be causal localization on semi-infinite space-like "strings" $x+\mathbb{R}_{+} e, e^{2}=-1$. It is easy to construct $m>0$ covariant string-localized fields $\Psi(x, e)$ in terms of semi-infinite line integrals on point-local fields [1]. The immediate advantage of such a construction is that one obtains two improvements in one stroke, the lowering of the short-distance dimension and the existence of a massless limit in terms of the two-point function. The simplest illustration is provided by the $d_{s d}=2$ point-local (pl) Proca vector potentials, which diverge as $m^{-1}$ for $m \rightarrow 0$, and its $d_{s d}=1$ string-local (sl) sibling whose two-point function turns out to possess a nontrivial massless limit.

The construction of free massive sl potentials in the same Hilbert space as their point-local counterparts guaranties that both belong to the same relative localization class ("Borchers class" [2]). They represent two different "field coordinatizations" of the same theory which, in the presence of interactions, implies that their particle content and S-matrix are identical.

\footnotetext{
1 Beware that strings in String Theory are not string-local in the sense of local quantum physics.
} 
The perturbative use of string-local elementary matter fields $\Psi(x, e)$ in Hilbert space QFT corresponds to formally gauge-invariant string-local composites in terms of pl fields in the Krein space setting of GT,

$\psi^{K}(x) e^{i g \int_{0}^{\infty} A_{\mu}^{K}(x+\lambda e) e^{\mu} \mathrm{d} \lambda}$

where the superscript $K$ refers to the pl indefinite metric Krein space setting of GT. Proposals to recover gaugeinvariant charge-carrying matter fields in terms of such formal expressions appeared already a long time before renormalization theory [3]. They played no role in the discovery of renormalized perturbation theory, but they reappeared later in Mandelstam's proposal to replace the perturbative gaugetheoretic setting of QED by one which uses solely Hilbert space compatible field strengths [4].

The construction of renormalized composites as (1) in GT is much harder than the perturbative construction of elementary sl fields in the setting in which these fields are elementary fields of renormalized perturbation theory. Steinmann confronted the difficult task of constituting (1) in every order of perturbative renormalized GT [5]. Morchio and Strocchi's (still ongoing) project is motivated by the problem to extract long-distance (infrared) information from GT by studying global limits within a positivity-restoring topology [6].

As already indicated, the present paper presents a new perturbation theory based on massive string-local vector potentials which are defined as semi-infinite space-like line integrals over field strengths. These covariant sl potentials are then directly used in the definition of the interaction density; they maintain the Hilbert space positivity and permit a smooth $m \rightarrow 0$ limit of their vacuum expectation values.

These fields are accompanied by new objects referred to as "escort" fields; they are sl $s=1$ scalar fields (not possible with pl field). They first appeared in the construction of sl intertwiners [1] as unexpected objects of curiosity; years later they reappeared as "escorts" of sl vector potentials in unpublished notes of Jens Mund (private communications) and it became clear that they play an important role in a linear relation between pl Proca fields and their sl siblings.

It turned out that these scalar escorts are indispensable objects in a perturbative Hilbert space setting of sl fields (SLFT) which only uses physical degrees of freedom. Far from being restricted to kinematical aspects of sl fields, they enter in a profound way into the dynamics; in some models they even appear already in the first order interaction density.

The appearance of the escort fields is particularly interesting in comparison with GT, where in order to uphold higher order gauge symmetry one is forced to enlarge the degrees of freedom by unphysical negative metric Stueckelberg fields and ghosts. Since they must be removed in the end by the imposition of gauge invariance, which cannot be achieved for all physically interesting objects, their role is reminiscent of catalyzers. ${ }^{2}$ In the SLFT setting one also has to introduce new fields (the escorts) but different from GT they do not introduce additional physical degrees of freedoms: they only use the already existing physical degrees of freedom and therefore do not have to be removed.

This is a new phenomenon in QFT, whereas in many body quantum mechanics rearrangements of degrees of freedom forming new fields are well known (e.g. the formation of bosonic Cooper pairs in superconductivity). These escort fields appear only in the presence of fields in a Hilbert space setting. This explains why they have not been seen in $s<1$; and since renormalizable $s \geq 1 \mathrm{pl}$ interactions exist only in a Krein space gauge setting there was no chance to encounter them.

In the new sl theory (referred to as SLFT) the lowest order perturbative interaction densities are defined in terms of Wick products of string-local covariant free vector potentials $A_{\mu}(x, e)$ (obtained by integrating point-local field strengths along space-like semi-lines) with point-local $s<1$ free matter fields. The higher order interactions then spread the string localization of the potentials to the matter fields and in this way force them to be sl without having to impose this as in (1). Interestingly they become much stronger sl than the $A_{\mu}$, which retain the linear relation with the $\mathrm{pl}$ field strengths.

These "sl-infected" elementary matter fields of the new SLFT perturbation theory are the Hilbert space counterpart of the composite Krein space fields (1). Whereas the sl vector potentials and the matter fields maintain their $d_{s d}$ (apart from higher order logarithmic corrections), that of matter fields coupled to a $d_{s d}=2$ pl Proca potential increases linearly with the perturbative order. It is the loss of predictive power as the result of the growth of undetermined parameters with the perturbative order which renders the direct perturbative use of the pl description (instead of viewing $\mathrm{pl}$ as a singular "field coordinatization" within the renormalizable sl formulation) unserviceable.

The short-distance behavior of gauge theories is similar to that of SLFT, but instead of being achieved in a natural way (maintaining positivity in the presence of interacting $s \geq 1$ fields through the weakening of localization) GT uses the brute force of cancellations between negative and positive metric Krein space contributions in intermediate states. This similarity of short distance limits supports the expectation that e.g. the asymptotic freedom short-distance property based on the behavior of the beta function which appears in the Callan-Symanzik equation is shared between GT and SLFT.

In the sl setting the fields $\Psi(x, e)$ remain of the Wightman type (bounded $d_{s d}$ ) except that the test function smearing must be extended to the $2+1$ dimensional de Sitter space

\footnotetext{
2 Perhaps the analogy to the magician's apprentice who is hardly able to control the ghosts is more realistic.
} 
of space-like directions $e$. The correlation functions of these sl matter fields are besides the sl vector potentials the only survivors in the $m \rightarrow 0$ QED limit in which, different from the massive case, pl fields do not even exist in the form of singular objects (Jaffe fields [7]). In view of this the absence of massless pl charge-carrying fields in QED is hardly surprising; in fact it is preempted on the basis of general arguments based on the quantum Gauss law [8].

The main problem of SLFT is not so much the transcription of the pl Wightman axiomatic [2], the hard problem is rather the extension of renormalization theory from pl to sl fields. The feasibility of such a project relies on the observation that sl fields have a better short-distance behavior than their $\mathrm{pl}$ counterparts. Whereas $d_{s d}$ of $\mathrm{pl}$ free tensor potentials increases with spin as $d_{s d}^{s}=s+1$, one can always construct an sl sibling with $d_{s d}^{s}=1$ independent of $s$ [20].

This implies that there is no problem to construct interaction densities $L$ for any spin within the power-counting bound $(P C B) d_{s d}^{\text {int }} \leq 4$ of renormalizability. But as in gauge theory where one has also to impose gauge invariance, the first order interaction density of SLFT must fulfill a physical condition which prevents total delocalization in the next and higher orders. Whereas gauge symmetry and gauge invariance have no natural relation with the principles of QFT, causal localization is directly tied to its foundations. The additional requirement is the so-called $L, V_{\mu}$ (or $L, Q_{\mu}$ ) pair condition, whose explanation needs the preparation of the next section.

A heuristic picture of its purpose is that of splitting a PCB violating first order pl interaction density $L^{P}$ involving only massive fields as $L^{P}=L-\partial V$ into a sum of a PCB obeying sl density $L$ and a sl divergence contribution $\partial^{\mu} V_{\mu}$ which carries the PCB violating contribution. Being a divergence in a model with a mass gap this term is expected to disappear in the adiabatic limit which expresses the perturbative S-matrix in terms of spacetime integrals over Minkowski space.

In this limit the S-matrix inherits the best of two worlds: the independence of the string direction $e$ from $L^{P}$ and the PCB-respecting $d_{s d}(L) \leq 4$ of the string-local interaction density. Since the construction of the $\mathrm{S}$-matrix is the first step in Bogoliubov's approach to the time-ordered vacuum expectation values of fields, one expects to be able to extend the $\mathrm{S}$-matrix construction to correlation functions of sl fields. In terms of Feynman diagrams the adiabatic limit corresponds to the integration over the $x^{\prime} s$ of inner propagator lines.

Similar to gauge invariance of the S-matrix, which is not a property of a single graph but rather the result of compensations between different contributions in the same perturbative order, one expects that the independence from the fluctuating "inner" $e$ 's cancels between different contributions, so that (in the case of correlation functions of fields) only the dependence of the correlated fields on their fluctuating $e^{\prime} s$ remains. These are the physical counterparts of the unphysical gauge-dependent fields.
This is similar to the operator formulation of BRST gauge invariance in [9], which in a more recent publication [10] has been referred to as causal gauge invariance (CGI). The significant conceptual difference is that the BRST gauge invariance of the $\mathrm{S}$-matrix $\mathfrak{s S}=0$ is in terms of an abstract global cohomological $\mathfrak{s}$ operation which has no relation to spacetime causal localization properties, whereas the $e$ independence is implemented as $d_{e} S=0$ within a differential form calculus in the $2+1$ dimensional de Sitter space of space-like directions $e, e^{2}=-1$. In this way higher spin interactions become re-integrated into the causal localization based QFT where positivity and localization are interdependent. SLFT is not a miracle but rather results from a clever use of existing ideas.

In the short-distance limit GT and SLFT behave in a similar way; in fact the beta functions of asymptotic freedom are expected to coalesce. It would be interesting to derive the Callan-Symanzik equation within SLFT and show there is no dependence on $e$. In contrast the correct description of long-distance properties (spacetime origin of infrared properties and in particular confinement) is expected to require the physical localization and positivity properties of SLFT, which is the extension of the principles of QFT to $s \geq 1$.

The new rules of SLFT only apply to interactions involving $s \geq 1$. It is not necessary and not even possible to use them for $s<1$; the attempt of using low spin ls fields leads to total delocalization which is incompatible with QFT and the use of $\mathrm{pl}$ for $s \geq 1$ leads to nonrenormalizability. There is only one QFT and the decision of whether to use pl or sl is not up to the working physicist but is fixed by the spin content of the interaction-defining fields.

Different from low spin pl interactions for which the first order PCB already implies the renormalizability in all orders, this is not necessarily the case in SLFT. An interesting illustration comes from self-interacting massive vector mesons. In that case the pair condition can be fulfilled but it induces a second order PCB violating $d_{s d}=5$ term which, if left uncompensated, would eliminate the self-coupled model from representing a QFT. First calculations indicate that the extension of the first order $L$ by an $A-H$ interaction leads to such a compensation and in this way saves the extended model. This, and not an alleged spontaneous symmetry breaking (SSB) "Higgs mechanism", would then be the raison d'étre for the $H$ just as in the calculation based on the BRST invariance of the S-matrix [9].

The use of $s>1$ sl fields in interaction densities which fulfill the first order pair property will certainly lead to PCB violating second order terms with even higher $d_{s d}$ unless one finds a compensating possibly extended field content involving lower spin fields. The realization of this picture for $s \geq 2$ interactions would amount to an enormous step into still dark corners of QFT of higher spin interactions. Higher spin fields for which this is not possible are not reactive within 
the setting of QFT. Being free fields associated to positive representation the lack of reactivity within QFT does not exclude the possibility that they couple to gravity through their conserved energy-momentum (E-M) tensor and cause backreactions to the gravitational field.

Calculations which could show whether a particular field is reactive or inert are laborious and SLFT is still very much in its beginnings. The only fields for which one can establish inertness are the sl fields associated to Wigner's massless infinite spin class; they already fail on the $L, V_{\mu}$ pair condition. If there is any physical role to play for such fields then it is as candidates for dark matter [11]. Unlike cold dark matter whose reduced reactivity and the possibility of its nonrelativistic description results from "coldness", this "Wigner stuff" is intrinsically inert.

Whereas the breakdown of $\mathrm{pl}$ renormalizability certainly does not mark the borderline between existing and nonexisting models of QFT, there are good reasons to believe that the SLFT renormalization requirements do precisely this. The fact that the perturbative series diverges and may only exist in the sense of a small coupling asymptotic expansion is not an argument against this idea. There are shortcomings which could explain this, for example the intrinsically singular nature of quantum fields used in the perturbative expansion.

In those few cases in which the existence has been established (integrable models in $d=1+1$ ) the construction starts from the other end, namely from the (in those models known) S-matrix and its connection with wedge-localized algebras of algebraic QFT. These existence proofs are based on the algebraic setting of QFT. In contrast to perturbation theory based on fields, these "top-down" constructions do not (yet?) arrive at generating fields for these algebras [12].

Returning again to more mundane problems, one may ask whether sl fields can play a role in the construction of energymomentum (E-M) tensors. In the existing literature most constructions of E-M tensors for free fields are presented in the setting of Lagrangian quantization, but it is well known that for $s \geq 1$ this leads to $\mathrm{pl}$ indefinite metric fields (and hence requires gauge theory). There is no problem in constructing E-M tensors in terms of positivity-obeying massive pl fields, but for $s>1$ these $\mathrm{pl} \mathrm{E-M}$ tensors have no massless limit, in agreement with a theorem by Weinberg and Witten [13].

Different from the statement in the No-Go theorem, which negates the existence of massless higher spin E-M tensors altogether, such tensors perfectly exist namely in the form of conserved sl tensors. The reason behind this is quite simple; it is a consequence of the nonexistence of positivity-preserving $s \geq 1$ massless tensor potentials and the fact that for $s \geq 2$ the E-M tensor cannot be expressed of the pl field strength (more in the next section). As sl tensor potentials, sl E-M tensors have the property that their $d_{s d}$ coalesce with their classical dimension in terms of mass units ("engineering dimension").
The strongest support for sl being the generic case of field localization in models of local observables (and pl the low spin exceptions) comes from a theorem of algebraic QFT. It states that in QFT with local observables and a mass gap particle can always be described in terms of operators which are localized in arbitrarily narrow space-like cones whose cores are linear semi-infinite space-like strings [14]. In a theory formulated in terms of (test-function-smeared) generating fields, these are sl fields with pl being a special case.

Being a structural theorem, it does not reveal in which models one needs sl instead of $\mathrm{pl}$ generating fields, but it excludes at least the need of having to use weaker than sl localized fields (e.g. space-like surfaces). The perturbative setting of SLFT answers this question by stating that all $s \geq 1$ interactions are SLFT, assuming that they fulfill the pair restriction and permit the preservation of higher order PCB through compensating field enlargement as previously explained.

Another point of foundational importance is to note that the only perturbative way to obtain a description of theories involving zero mass $s \geq 1$ tensor potentials is in the form of massless limits of correlation functions. The construction of the Hilbert space and the operators acting in it (if needed) follows from Wightman's reconstruction theorem [2].

The conceptual simplicity of a theory in a Wigner-Fock Hilbert space is lost in the massless limit (with the mass as a natural infrared cutoff). This already happens in the case of the massless limit of free massive two-point functions of $s \geq 1$ sl potentials, where the reconstruction leads to the more involved $m=0$ helicity representation. The massless limit of the gauge-dependent pl matter fields in the same Krein space as their massless counterpart is simpler but contains less physical information.

The new interest in sl fields can be traced back to the solution of the localization properties of Wigner's zero mass infinite spin representation class $[1,15]$ in terms of the modular localization theory of algebraic QFT (a historic/philosophical view can be found in [16]). But the construction of finite spin sl fields and their use in a new perturbative setting does not require knowledge about modular localization. A first account of their potential use has been given in [17]. A shorter publication which addresses similar problems to those in the present paper appeared [18].

Looking at axiomatic QFT in the sense of Wightman, it seems that the postulates of a point-local QFT can be rewritten in terms of sl Wightman fields $\Psi(x, e)$ for which the smearing functions are Schwartz functions in the $d=1+2 \mathrm{de}$ Sitter space extended Minkowski space. The analytic properties of vacuum expectation values should be related to those of the intertwiners in [1] and the basic structural theorems (TCP, Spin\&Statistics, etc.) are expected to follow.

As previously mentioned, the perturbative string-local field theory (SLFT) shares many formal similarities with 
gauge theory (GT). This is particularly evident if one formulates GT not in terms of a functional setting pictured by Feynman diagrams, but instead uses the so-called causal gauge-invariant operator setting (CGI) [9]. The reason is that gauge invariance holds only on-shell which makes it difficult to describe properties as positivity (unitarity) graphically.

In particular it is impossible to distinguish between offshell Mexican hat SSB setting and the on-shell induction of Mexican hat like $H$ self-interacting from the second order BRST gauge invariance $\mathfrak{s} S=0$ of the S-operator. In the CGI operator formulation of the BRST gauge formalism $[9,10,19]$ there is a clear-cut distinction, whereas in the SLFT setting the idea of an SSB would not even arise since the matter fields which couple to sl vector mesons become themselves sl and hence do not comply with a physical picture of a global symmetry breaking in which the local current remains conserved but the global charge diverges.

The content of the five sections is as follows.

The next section presents the kinematics of SLFT i.e. relations between the pl potentials and their sl counterpart in which the escorts appear for the first time. It also explains the physics behind the first order $L, V_{\mu}$ pair requirement.

In the third section it is shown that even in the absence of interactions the causal localization which GT formally attributes to a Wilson loop is not identical with that in the Hilbert space setting (breaking of Haag duality but preservation of Einstein causality).

Some second order perturbative results and their interpretation are presented in Sect. 4.

Section 5 presents formal analogies with the CGI operator formulation of the BRST formalism.

The outlook contains comments about ongoing calculations and conjectures about what one hopes to accomplish in the future.

Many of the ideas arose in extensive discussions over several years with Jens Mund, but the responsibility for possible errors and less than perfect presentations rest on my shoulders.

It should be mentioned that within this shared project of SLFT there are two forthcoming articles by Mund. Whereas the present paper accounts for conceptual changes and the resulting different way in which seemingly well-established past results appear in the new light, Mund's contributions address the deeper mathematical problems and also contain the first attempts to go beyond the S-matrix and face the problem of correlation functions of interacting sl fields [20,21].

\section{A Hilbert space alternative to local gauge theory, kinematical aspects}

Massive free spin $s \geq 1$ fields are commonly described in terms of degree $s$ tensor potentials. For $s=1$ this reduces to the well-known Proca potential

$$
\begin{aligned}
A_{\mu}^{P}(x)= & \frac{1}{(2 \pi)^{3 / 2}} \int\left(e^{i p x} \sum_{s_{3}=-1}^{1} u_{\mu}\left(p, s_{3}\right) a^{*}\left(p, s_{3}\right)\right. \\
& + \text { h.c. }) \frac{\mathrm{d}^{3} p}{2 p_{0}}
\end{aligned}
$$

$\left\langle A_{\mu}^{P}(x) A_{\nu}^{P}\left(x^{\prime}\right)\right\rangle=\frac{1}{(2 \pi)^{3}} \int e^{-i p \xi} M_{\mu \nu}(p) \frac{\mathrm{d}^{3} p}{2 p_{0}}$,

$M_{\mu \nu}=-g_{\mu \nu}+\frac{p_{\mu} p_{v}}{m^{2}}$

To this point-local field one may associate two stringlocal fields, a vector potential $A_{\mu}(x, e)$ and a scalar field $\phi(x, e)[20]$, as well as two "field-valued differential forms" in $e$-space namely a one-form $u(x, e)$ and a two-form $\hat{u}(x, e)$

$$
\begin{aligned}
A_{\mu}(x, e) & =\int_{0}^{\infty} F_{\mu \nu}(x+\lambda e) e^{\nu} \mathrm{d} \lambda, \quad \text { with } F_{\mu \nu}(x) \\
& =\partial_{\mu} A_{\nu}^{P}(x)-\partial_{\nu} A_{\mu}^{P}(x), \\
\phi(x, e) & =\int_{0}^{\infty} A_{\mu}^{P}(x+\lambda e) e^{\mu} \mathrm{d} \lambda, \quad e^{2}=-1 \\
u & =d_{e} \phi=\partial_{e^{\mu}} \phi d e^{\mu}, \quad \hat{u}=d_{e}\left(A_{\alpha} d e^{\alpha}\right)
\end{aligned}
$$

which are all members of the equivalence class of relatively string-local fields which are associated to the point-local $A_{\mu}^{P}$ Proca field (the sl "Borchers class"). It is well known [2] that, in the presence of a mass gap, interacting point-local fields within the same localization class lead to the same physics (particles, S-matrix); this continues to be valid for string-local fields $[14,22]$.

Throughout this paper the differential form calculus on the $d=1+2$ de Sitter space of string-directions will play an important role. As the $x$, the $e^{\prime} s$ are variables in which the fields fluctuate; they bear no relation with the "mute" gauge-fixing parameters of GT.

String-local vector potentials fulfill $e^{\mu} A_{\mu}(x, e)=0$, and in the massless limit also $\partial^{\mu} A_{\mu}(x, e)=0$. These relations are not imposed gauge conditions but rather intrinsic properties of string-local potentials which result from the above definitions. Note that only $A_{\mu}$ possesses a zero mass limit. Whereas $A_{\mu}^{P}$ has a $m^{-1}$ mass divergence, $u$ and the difference of two $\phi$ with different $e$ are logarithmically divergent just as the free field in $d=1+1$ whose exponentials lead to new superselection sectors (Sect. 3 (25)). Although all sl fields are free fields, their common origin from the pl Proca potential leads to mixed two-point functions (see below).

One may change the string "density" $\mathrm{d} \lambda \rightarrow q(\lambda) \mathrm{d} \lambda, q(\infty)$ $=1$ within the linear field class. There is no conceptual problem with this continuous enlargement since quantum fields, in contrast to classical fields, have no observable "individuality". The latter property is an attribute of particles which share their superselected charges with their associated field-classes 
with which they are (large-time) asymptotically related [22]. Renormalizability requires one to define PCB interaction densities in terms of maximally fourth degree polynomials of fields with the lowest short-distance dimension within a field class.

In order to obtain linear relations between these fields as

$$
A_{\mu}=A_{\mu}^{P}+\partial_{\mu} \phi
$$

one must use the same $q(\lambda)$ and in order to maintain simplicity of two-point function

$$
\begin{gathered}
\left\langle A_{\mu}(x, e) A_{\mu^{\prime}}\left(x^{\prime}, e^{\prime}\right)\right\rangle \\
=\frac{1}{(2 \pi)^{3}} \int e^{-i p\left(x-x^{\prime}\right)} M_{\mu \mu^{\prime}}^{A, A}\left(p ; e, e^{\prime}\right) \frac{\mathrm{d}^{3} p}{2 p_{0}} \\
M_{\mu \mu^{\prime}}^{A, A}\left(p ; e, e^{\prime}\right)=-g_{\mu \mu^{\prime}}-\frac{p_{\mu} p_{\mu^{\prime}}\left(e \cdot e^{\prime}\right)}{(p \cdot e-i \varepsilon)\left(p \cdot e^{\prime}+i \varepsilon\right)} \\
\quad+\frac{p_{\mu} e_{\mu^{\prime}}}{(p \cdot e-i \varepsilon)}+\frac{p_{\mu} e_{\mu^{\prime}}^{\prime}}{\left(p \cdot e^{\prime}+i \varepsilon\right)} \\
M^{\phi \phi}=\frac{1}{m^{2}}-\frac{e \cdot e^{\prime}}{(p \cdot e-i \varepsilon)\left(p \cdot e^{\prime}+i \varepsilon\right)}, \\
M_{\mu}^{A \phi}=\frac{1}{i}\left(\frac{e_{\mu}^{\prime}}{p \cdot e^{\prime}+i \varepsilon}-\frac{p_{\mu} e \cdot e^{\prime}}{(p \cdot e-i \varepsilon)\left(p \cdot e^{\prime}+i \varepsilon\right)}\right)
\end{gathered}
$$

These expressions, which first appeared in unpublished notes by Jens Mund, may be either directly derived from the above line integral or be obtained from the $A, A^{P}$ and $\phi$ intertwiners ${ }^{3}$ (the $e$-dependent denominators including the $\varepsilon$-prescription result from the Fourier transformation of the Heaviside function). Clearly the $\lambda$-integration (3) has lowered the short-distance dimension of the vector potential from $d_{s d}^{P}=2$ to $d_{s d}=1$ and the heuristic reading of (4) is that the derivative $\partial \phi$ of the $d_{s d}=1 \phi$-escort removes the most singular part of $A^{P}$ at the price of directional $e$-fluctuation. In the massless limit $A^{P}$ as well as $\phi$ diverges, but the string-local potential $A_{\mu}(x, e)$ remains well defined; its four-dimensional curl is the point-local field strength.

The necessity to work with string-local potentials and the appearance of mixed two-point functions of free fields is the price for working in the Hilbert space of physical degrees of freedom. In contrast the indefinite metric Krein space of gauge theory contains in addition to the Gupta-Bleuler and Stückelberg indefinite metric degrees of freedom also those of "ghost" fields. In this case there are no mixed contributions since these unphysical free fields have independent degrees of freedom. The BRST gauge formalism permits to obtain some physical data, but falls short of extracting a full QFT.

\footnotetext{
3 A systematic and detailed account of the construction of string-local fields and their use in a string-extended Epstein-Glaser construction of time-ordered products can be found in [21].
}

The sl $A_{\mu}(x, s)$ and its scalar "escort" $\phi(x, e)$ are different fields, but they do not enlarge the degrees of freedom; in fact they are linear in the same Wigner creation and annihilation operators $a^{\#}\left(p, s_{3}\right)$ of the point-local Proca potential. Scalar $s=1$ fields exist only in the form of massive stringlocal fields [1]. Although in the present context they do not add the degrees of freedom, their additional appearance in interaction densities plays an important role in upholding string localization of higher order interacting fields. Textbook QFT only uses pl Wightman field; these are the only fields which are in the range of Lagrangian quantization but their use in positivity-maintaining interactions is limited to low spin $s<1$ fields. QFT of $s \geq 1^{4}$ requires the use of sl fields and each such field is accompanied by $s$ lower spin sl escorts (see below). The nonperturbative mathematical tools which lead to the famous theorems of local quantum physics (TCP, Spin\&Statistics, etc.) require the presence of positivity (unitarity).

The reader will encounter many new concepts but can be assured that all of them result in a natural way from reconciling higher spin interactions with the causal localization principles in the Hilbert space setting of QT. In particular the existence of massless vector- (more general $s>1$ tensor) potentials in Hilbert space is tied to the existence of the $m \rightarrow 0$ limit of sl massive correlation functions. It is precisely on the level of correlation functions were operators belonging to different Wigner representation classes (massive and massless $s \geq 1$ representations) coalesce. Even more important is their role in a future spacetime understanding of an operator formalism and the replacement of the Wigner-Fock Hilbert space in the infrared regime (infraparticles, confinement).

Equation (4) resembles an operator gauge transformation. But there is no gauge freedom in a positivity maintaining description; it rather expresses the relation between free $\mathrm{pl}$ and sl vector potentials. It should be maintained in the presence of interactions with a complex matter field. Whereas the interaction density is defined in terms of a free pl matter field $\psi_{0}(x)$, the interaction with sl potentials will convert these fields into an interacting sl field $\psi(x, e)$. One also expects that this sl field has a very singular interacting pl sibling $\psi^{P}$ (the analog of the $\mathrm{pl}$ fields in the nonrenormalizable $\mathrm{pl}$ Hilbert space setting) and that both are related (in the sense of normal products between interacting fields) as ${ }^{5}$

$$
\psi^{P}(x)=e^{-i g \phi(x, e)} \psi(x, e)
$$

This together with (4) is certainly reminiscent of a gauge transformation of a matter field interacting with a vector

\footnotetext{
4 Unless otherwise stated, the terminology "QFT" refers to a Hilbert space setting i.e. does not include GT.

5 I am indebted to Jens Mund for informing me that this relation has meanwhile been checked in lowest nontrivial order of massive QED.
} 
potential. But in the present context it represents a relation between two "field coordinatizations" of the same theory, one being a string-local Wightman field (bounded $d_{s d}$ ) and the other a field with unbounded short-distance dimension as known from nonrenormalizable couplings [23]. This is a class of fields which are too singular (unbounded $d_{s d}$ ) in order to be compactly localizable in the sense of Wightman [2]; such fields have been studied by Jaffe [7] who illustrated his more singular fields in terms of Wick-ordered exponentials of free fields $\exp \varphi$.

After this brief excursion into uncharted territory, this section returns to the kinematic aspects of sl free fields.

The formal similarity of the directional variable $e$ of stringlocal fields with a (noncovariant) "axial" gauge parameter should not hide the fact that its gauge-theoretic interpretation $^{6}$ caused unsolvable short- and long-distance problems, which finally led to its abandonment. The reason behind this failure is that fluctuations in the $d=1+2$ the unit Sitter space of space-like directions in individual string-local fields cannot be reconciled with a gauge interpretation; fortunately what was a curse in the use as an axial gauge turns out to be a blessing in the Hilbert space setting of $s=1$ interactions.

This construction permits a generalization to any integer spin [20]. Massive free fields of spin $s$ and short-distance dimension $d_{s d}^{s}=s+1$ are conveniently described in terms of symmetric point-local potentials $A_{\mu_{1} \cdots \mu_{s}}^{P}$ of tensor degree $s$. A corresponding string-local tensor with $d_{s d}^{s}=1$ can be obtained in analogy to the vector potential with the help of repeated semi-infinite line integrals

$$
\begin{aligned}
\phi_{\mu_{1}, \ldots, \mu_{k}}(x . e)= & \int_{0}^{\infty} \cdots \int_{0}^{\infty} \mathrm{d} \lambda_{k+1} \cdots \mathrm{d} \lambda_{s} e^{\mu_{k+1}} \cdots e^{\mu_{s}} \\
& \times A_{\mu_{1}, \mu_{2}, \ldots, \mu_{k}, \ldots, \mu_{s}}^{P}\left(x+\lambda_{k+1} e+\cdots \lambda_{s} e\right)
\end{aligned}
$$

For the sake of simplicity of notation we specialize to $s=2$ in which case the corresponding relation to (4) is

$$
\begin{aligned}
g_{\mu_{1} \mu_{2}}(x, e)= & g_{\mu_{1} \mu_{2}}^{P}(x)+\operatorname{sym} \partial_{\mu_{1}} \phi_{\mu_{2}}(x, e) \\
& +\partial_{\mu_{1}} \partial_{\mu_{2}} \phi(x, e)
\end{aligned}
$$

where our notation pays tribute to the fact that the metric tensor of general relativity is the principle physical candidate for a symmetric second degree tensor. Note that in this case the string-local field has 2 string-local escorts, a scalar $\phi(x, e)$ and a vector $\phi_{\mu}(x, e)$. By construction the symmetric tensor fulfills $e^{\mu_{1}} g_{\mu_{1} \mu_{2}}=0$ and for $m=0$ the identity $\partial^{\mu} g_{\mu \nu}=0$ (as $\partial^{\mu} A_{\mu}(x, e)=0$ this is not a gauge condition).

The field strength associated to the symmetric $g_{\mu \nu}$ tensor field of $g_{\mu \nu} s=2$ field of degree 4, which has the same mixed symmetric-antisymmetric permutation symmetry as

\footnotetext{
6 The gauge-theoretic $e$ is considered to be "mute" i.e. it is the same for all gauge fields and remains unaffected by Lorentz transformations.
}

the Riemann tensor of relativity (the "linearized Riemann tensor") is

$R_{\mu \nu \kappa \lambda}(x)=\frac{1}{2}$ as $\partial_{\mu} \partial_{\kappa} g_{v \lambda}$

where the antisymmetrization as takes place between the two symmetric pairs. This $R$-tensor, which is the $s=2$ analog of the $s=1$ field strength $F_{\mu \nu}$, is the lowest $d_{s d}$ point-local massless $s=2$ field (the antisymmetrization lowers the $d_{s d}$ from 4 to 3 ).

The extension of (4) and (8) to spin $s>2$ should be clear: a massive point-local degree $s$ tensor potential corresponds to a string-local potential of the same tensor degree and " $\phi$ escorts" of lower tensor degree of which only the string-local degree $s$ potential has a massless limit. The lowest degree point-local tensor field which permits a massless limit is a field strength of degree $2 s$ and short-distance dimension $d_{s d}=s+1$. In analogy to (9), it results from the application of $s$ derivatives to a tensor of degree $s$ and subsequent antisymmetrization between the two sets of indices.

The intertwiner $u(p, s)$ for massive point-local tensor fields of degree $s$ and short-distance dimension $d_{s d}=s+$ 1 relates the $2 s+1$ spin space with the space of symmetric covariant tensor. They are divergence free (as $A_{\mu}^{P}$ ) and traceless. It is simpler to calculate their momentum space two-point functions which consists of linear combinations of tensors of degree $2 s$ formed from the Minkowski spacetime $g_{\mu \nu}$ and products of $p_{\mu}$ made dimensionless by multiplication with appropriate inverse mass powers. The requirement of vanishing trace and divergence determines the two-point function up to a numerical factor.

These constructions for general spin lead to $s>2$ expressions for the energy-momentum tensor. Besides the pl E-M tensor there is an sl tensor. They are expected to describe the same charges (generators of the Poincaré group) but their $m \rightarrow 0$ limit behavior is quite different. The $\mathrm{pl}$ tensors have no massless limit for $s>1$, whereas their sl counterparts continue to exist for $m=0$. As already mentioned in the introduction this is quite interesting in view of the WeinbergWitten No-Go theorem for massless $s \geq 2$ E-M tensors [13]. These authors tacitly assumed pl localization. The theorem is hardly a surprise since the massless $\mathrm{pl}$ free fields from which they are formed cease to exist for $s \geq 1$ and the $s=1$ case only works because the corresponding E-M tensor can be written in terms of the field strength. The construction of the sl E-M tensor is presented in [11].

The $d=1+2$ directional de Sitter space impart these string-local fields (and related field-valued differential forms) with a rich differential-geometric structure which plays an important role in the new positivity-maintaining SLF perturbation theory. In this setting all fields are physical; the gauge-invariant local observables correspond to point-local (generally composite) fields, whereas the interacting vector 
potentials and the matter fields $\Psi(x, e)$ are string-local generalizations of Wightman fields (polynomially bounded in momentum space or equivalently $d_{s d}<\infty$ ).

A surprising collateral kinematic result of this observation is the statement (easy to verify) that the angular averaging over $e$ within a space-like plane leads to the Coulomb (or radiation) vector potential. It is well known that it acts in a Hilbert space and that its lack of covariance makes it unsuitable for renormalized perturbation theory. The fact that it results from directional averaging of covariant string-local potential (which plays the central role in the new covariant SLFT Hilbert space setting) may come as a surprise to some.

This suggests to construct perturbative results in Coulomb "gauge" by first working in the covariant SLFT formulation and afterwards applying the rotational averaging instead of trying to perform renormalized perturbation theory directly in the CG setting (which seem to be an impossible task).

Before taking up the issue of interactions, it is interesting to compare the SLFT setting with the BRST gauge formalism. The latter is based on a the action of a nilpotent $\mathfrak{s}$-operation on indefinite metric fields in a Krein space ( $K=$ Krein) extended by "ghost operators". In the notation of [9] it reads

$$
\begin{aligned}
& \mathfrak{s} A_{\mu}^{K}=\partial_{\mu} u^{K}, \quad \mathfrak{s} \phi^{K}=u^{K}, \quad \mathfrak{s} \hat{u}^{K}=-\left(\partial A^{K}+m^{2} \phi^{K}\right) \\
& \mathfrak{s} B:=i[Q, B], \quad Q=\int \mathrm{d}^{3} x\left(\partial^{v} A_{v}^{K}+m^{2} \phi^{K}\right) \overleftrightarrow{\partial}_{0} u^{K}
\end{aligned}
$$

$Q$ is the so-called ghost charge (associated to a conserved ghost current) whose properties ensure the nilpotency $\left(\mathfrak{s}^{2}=\right.$ 0 ) of the BRST $\mathfrak{s}$-operation. The $A_{\mu}^{K}$ is a point-like massive vector meson in the Feynman gauge and $\phi^{K}$ is a free scalar field whose Krein space two-point function has the opposite sign (a kind of negative metric scalar Stückelberg field). The "ghosts" $u, \hat{u}$ are free "scalar fermions" whose presence is necessary in order to recover the perturbative positivity of local observables and a unitary S-matrix.

The s-invariance of the scattering $S$-operator in gauge theory and the $d_{e}$-independence in the string-local setting are both related to cohomology; but whereas the former has no relation to spacetime, the $d_{e}$ acts on the space-like string directions of the in $e$ independently fluctuating fields.

Since there are string-local fields with $d_{s d}=1$ for all spins, there also exist string-local interaction densities within the power-counting limit $d_{s d}^{\text {int }}=4$. But as already mentioned in the introduction, there is another physical restriction, which has no counterpart in the point-local case: couplings of string-local fields are only physical if their higher order extensions preserve string localization and if there remains a subalgebra of pointlike generated local observables.
This requirement, which plays no role within the pointlocal renormalization formalism, severely restricts interactions involving sl fields so that some of the advantage of low short-distance dimensions is lost. In the following this will be illustrated in three examples involving massive vector mesons (all fields are free fields). The first two models describe a massive vector meson which couples either with a complex scalar field $\varphi$ (scalar "massive QED") or with a Hermitian scalar field $H$,

$L^{P}=g A_{\mu}^{P} j^{\mu}, \quad j_{\mu}=\varphi^{*} \overleftrightarrow{\partial}{ }_{\mu} \varphi:$

$L^{P}=g m A_{\mu}^{P} A^{P, \mu} H$

where the mass factor $m$ accounts for the mass dimension ("engineering dimension") of the interaction density. In both cases $A_{\mu}^{P}$ is the point-local $d_{s d}=2$ Proca potential so that the point-local interaction density $L^{P}$ violates the PCB restriction of renormalizability.

Using the relation between the Proca potential and its string-local counterpart $A_{\mu}$ including its escort field $\phi$ (4), one may rewrite the nonrenormalizable point-local interactions (11) into a $d_{s d}^{\text {int }} \leq 4$ string-local expression plus the divergence of another operator $V_{\mu}$.

$$
\begin{aligned}
& L^{P}=L-\partial^{\mu} V_{\mu}, \\
& L=g A_{\mu} j^{\mu}, \quad V_{\mu}=j_{\mu} \phi,
\end{aligned}
$$

The second line presents the wanted pair $L, V_{\mu}$ for massive scalar QED, for both operators $d_{s d}=4$. The renormalizationpreventing point-local interaction density $d_{s d}\left(L^{P}\right)=5$ has been separated into two string-local contributions in such a way that the renormalizability spoiling $d_{s d}=5$ contribution has been collected into the divergence of $V_{\mu}$. In massive QFTs such divergence terms may be disposed of in the adiabatic limit so that the first order S-matrix of the powercounting violating $L^{P}$ is the same as that of its better behaved string-local counterpart $L$. Although far from obvious, this idea of disposing renormalizability-violating terms at infinity can be generalized to higher orders. It is not limited to the S-matrix, but it also leads to the construction of a polynomial bounded correlation function of string-local quantum fields (private communication by Jens Mund). In other words the sl perturbation theory complies with the localization properties of string-local Wightman fields.

There is widespread belief that for perturbative renormalization theory one needs (either canonical or functional integral) Lagrangian quantization. But this is not correct; even for $\mathrm{pl}$ perturbation theory one only needs a scalar interaction density $L^{P}$ in terms of free fields. These free fields need not be Euler-Lagrange fields; rather any free field obtained from covariantization of Wigner's pure quantum unitary representation theory of the Poincaré can be used. In fact, Lagrangians for most higher spin fields are not known and sl fields are never Euler-Lagrange. The Stückelberg- 
Bogoliubov-Epstein-Glaser perturbation theory is based on the causal iteration of the first order scalar interaction density $L$ made from local Wick-products of free fields. There are no infinities, but the iteration leads to a growing number of new parameters (counterterm parameters) whose number only remains finite in the case of the $\mathrm{PCB} d_{s d}(L) \leq 4$.

The $L, V_{\mu}$ pair for the $H$ coupling is less simple ${ }^{7}$ since now also $L$ depends on $\phi$

$$
\begin{aligned}
& L=g m\left(A \cdot A^{P} H+\phi A \cdot \partial H-\frac{m_{H}^{2}}{2} \phi^{2} H\right), \\
& V^{\mu}=g m\left(\phi A_{\mu}^{P} H+\frac{1}{2} \phi^{2} \partial^{\mu} H\right) .
\end{aligned}
$$

In this case the verification of the identity (13) requires the use of the free field equation for $H$ with mass $m_{H}(m=$ mass of vector meson).

As Mund has shown (unpublished) a similar $L, \partial V$ pair exists for self-interacting massive vector mesons, e.g.

$$
\begin{aligned}
L^{P} & =\sum \varepsilon_{a b c} F_{a}^{\mu \nu} A_{b, \mu}^{P} A_{c, v}^{P}=L-\partial V, \\
L & =\sum \varepsilon_{a b c}\left\{F_{a}^{\mu \nu} A_{b, \mu} A_{c, v}+m^{2} A_{a, \mu}^{P} A_{b}^{\mu} \phi^{c}\right\} \\
V_{\mu} & =\sum \varepsilon_{a b c} F_{a}^{\mu \nu}\left\{A_{b, v}+A_{b, v}^{P}\right\} \phi_{c} .
\end{aligned}
$$

For the verification one again has to use the field equation, which in this case reads $\partial^{v} F_{\mu \nu}=m^{2} A_{\mu}^{P}$.

For the extension to higher orders it is helpful to express the point-local nature in terms of the differential form calculus on a de Sitter space,

$d_{e}(L-\partial V)=0$.

The existence of such pairs with $L$ within $d_{s d}^{\text {int }} \leq 1$ turns out to be the prerequisite for the existence of local observables within a string-local setting. They also prevent the higher order total delocalization over all of spacetime. It should, however, be emphasized that the construction of $L, V_{\mu}$ pairs is a problem which can be pursued independent of $L^{P}$. Whether a collection of free fields permits a (maximally quadrilinear) coupling $L$, which can be completed to a $L, V_{\mu}$ pair is a well-defined mathematical problem within the setting of differential forms on $d=1+2$ de Sitter space. Whereas $L$ must stay within PCB, the $d_{s d}$ of $V_{\mu}$ may have contributions above $d_{s d}=4$; as long as these contributions do not lead to higher order short-distance contributions to $L$ beyond $d_{s d}=4$ the model remains sl renormalizable.

The exactness of the zero form $L-\partial V$ in (16) is a rather restrictive localization requirement. Such pairs within the power-counting bound for $L$ turn out to be unique (in case they exist) modulo additive changes of $V_{\mu}$ terms with vanishing divergence. Since in massive models the divergence

\footnotetext{
7 The perturbative calculations are simpler if one replaces only as many $A^{P}$ by $A$ as needed to obtain $d_{s d}(L)=4$.
}

$\partial V$ disappears in the adiabatic on-shell limit, the first order contribution to the S-matrix are equal

$S^{(1)}=\int L^{P}=\int L$

String-local $L, V_{\mu}$ pairs are the starting point for the perturbative construction of the $e$-independent S-matrix and correlation functions of string-local fields. The problem how to maintain string localization in higher order perturbations is closely related to the problem of preserving the $e$-independence of the S-matrix. This leads to a normalization condition on higher order time-ordered products of $L-\partial V$, which will be commented on in Sect. 4 .

Note that the $L, V$ formalism is not directly applicable to $m=0$, since $\phi$ and $V_{\mu}$ have no massless limit. Behind this formal problem there is a radical conceptual change (breakdown of Wigner-Fock particle Hilbert space, infraparticles in QED, QCD confinement) whose spacetime implications have remained outside of our conceptual understanding of QFT. In fact these problems are outside the physical range of gauge theory; whereas short-distance properties of unphysical gauge-dependent fields are believed to share their asymptotic short-distance behavior with those of their sl physical counterparts (in particular the QCD asymptotic freedom) one does not expect that problems related to confinement can be accounted for in gauge theory. Here the long-distance fluctuations of string directions will be important. Following Wightman's reconstruction theorem [2] the massless QFT should be reconstructed from the massless limit of the massive correlation functions thus avoiding direct questions concerning the fate of the Wigner-Fock particle space.

\section{Wilson loops, Haag duality and the Aharonov-Bohm effect}

The following section extends ideas which were already presented in [18].

Consider the space-like Wilson loop for a string-local vector potential. In the massive case one obtains from (4)

$$
\begin{aligned}
\oint A_{\mu}(x, e) \mathrm{d} x^{\mu} & =\oint\left(A_{\mu}(x)+\partial_{\mu} \phi(x, e)\right) \mathrm{d} x^{\mu} \\
& =\oint A_{\mu}(x) \mathrm{d} x^{\mu}, \quad m>0,
\end{aligned}
$$

whereas in the massless limit the separate contributions to the integrand diverge and instead one finds

$$
\begin{aligned}
& \oint\left(A_{\mu}(x, e)-A_{\mu}\left(x, e^{\prime}\right)\right) \mathrm{d} x^{\mu} \\
& =\oint \partial_{\mu}\left(\phi(x, e)-\phi\left(x, e^{\prime}\right)\right) \mathrm{d} x^{\mu}=0 \text { for } m=0 .
\end{aligned}
$$


It is important to notice that, although neither $\phi$ nor $\partial_{\mu} \phi$ possess massless limits, the mass singularities cancel in the difference between $\phi^{\prime}$ s with different $e$-directions. This can be seen either in terms of the $e$-dependence of the intertwiners or by using the fact that the $m^{-2}$ in (5) cancel in the 2-point function,

$$
\begin{aligned}
& \left\langle\psi\left(x ; e_{1}, e_{1}^{\prime}\right) \psi\left(x ; e_{2}, e_{2}^{\prime}\right)\right\rangle, \\
& \psi\left(x ; e, e^{\prime}\right):=\phi(x, e)-\phi\left(x, e^{\prime}\right) .
\end{aligned}
$$

The $e$-independence of the loop integral despite its $e$ dependent integrand is reminiscent of its gauge invariance in the Krein space setting of point-local vector potentials. Later we will return to this analogy.

For the following it is convenient to work with operators instead of singular quantum fields. A regularization of the vector potential in terms of a convolution with a smooth function $f$, which is localized around a small ball $B$ at the origin, leads to the regularized loop operator

$$
\oint A_{\mu}^{\mathrm{reg}}(x, e) \mathrm{d} x^{\mu}, \quad A_{\mu}^{\mathrm{reg}}(x, e):=\int f\left(x-x^{\prime}\right) A_{\mu}\left(x^{\prime}, e\right) \mathrm{d}^{4} x^{\prime} .
$$

It commutes with all operators whose localization region $\mathcal{O}$ is such that there exists a direction $e$ for which the regularized half-cylinder does not intersect $\mathcal{O}$. This includes in particular all convex regions which do not intersect the torus $l^{\text {reg }}$, which results from regularizing the loop $l$.

Operators whose localization region is such that there exists no choice of $e$ which permits one to avoid an intersection with the regularized semi-infinite cylinder $l^{\mathrm{reg}}+\mathbb{R}_{+} e$ do not commute with the regularized Wilson loop. This includes in particular operators which are localized in a torus which loops through $l^{\text {reg }}$ without intersecting it.

By allowing $e$ to vary along the Wilson loop such that $e(\alpha)$ moves through a loop in the directional de Sitter spaces as $x(\alpha)$ sweeps through the Wilson loop, one enlarges the possibilities of avoiding intersections; but in the case of a torus which intertwines $l^{\text {reg }}$ without touching, an intersection with the $e$-extended Wilson loop is unavoidable. The dependence on $e$ is "topological"; the Wilson loop "remembers" that its integrand had a directional dependence but it forgets in which direction it pointed.

This problem can be investigated directly in terms of the localization property of the electromagnetic field strength $F_{\mu \nu}$ without using vector potentials [24]. The result is that the operator representing a regularized magnetic flux through a surface $D$ does not change under deformations of $D$ as long as its boundary $\partial D$ stays the same. Any operator which is localized in a contractible region outside the regularized torus $\partial D+B$ commutes with the flux operator but, as shown in [24], there are operators associated with interlocking but not intersecting toroidal regions which do not commute with the regularized magnetic flux operator. The authors refer to such a situation as the "breakdown of Haag duality".

Recall that Einstein causality states that two operators commute if their localization regions are space-like separated. In terms of operator algebras this means

$\mathcal{A}(\mathcal{O}) \subseteq \mathcal{A}\left(\mathcal{O}^{\prime}\right)^{\prime}, \quad$ Einstein causality,

$\mathcal{A}(\mathcal{O})=\mathcal{A}\left(\mathcal{O}^{\prime}\right)^{\prime}$, Haag duality,

where the dash on the region refers to the causal complement and that on the operator algebra to its commutant. Our intuitive understanding of causal localization is however in terms of Haag duality [22]; we expect that an operator which commutes with all algebras which are localized in the causal complement of a region $\mathcal{O}$ is really localized in $\mathcal{O}$ i.e. is a member of $\mathcal{A}(\mathcal{O})$.

Haag duality holds for all algebras which are generated by massive free fields and is believed to remain valid for observable subalgebras localized in multiply connected regions. But the properties of magnetic fluxes in QED show that Haag duality is violated for multiply connected subalgebras generated by point-local $s \geq 1$ massless field strengths $\left(F_{\mu \nu}, R_{\mu \nu \lambda \kappa}, \ldots\right)$. In other words, there are operators in $\mathcal{A}\left(\mathcal{O}^{\prime}\right)^{\prime}$ which do not belong to $\mathcal{A}(\mathcal{O})$ and the Wilson loop operator with its topological $e$-dependence is an example. Interestingly these violations of Haag duality happen in theories in which the potentials are necessarily stringlocal.

The "quirky" feeling that there may be some problems with causality in the A-B effect ${ }^{8}$ has its origin in the naive identification of the gauge-theoretic quantum causality in Krein space with that of a QFT in Hilbert space. The use of $\mathrm{pl}$ vector potentials in Krein space is not incorrect; but it carries the danger of identifying the Wilson loop as an object localized on a circle. The ls setting is safe in this respect since the topological memory of the $e$ dependence is precisely what one needs to be reminded that, although the Wilson loop object commutes with all operators localized in the causal complement of the torus (the thickened Wilson loop), it is not localized on it. This is what the violation of Haag duality in Einstein-causal QFT means. It occurs in all models involving massless $s \geq 1$ fields.

The violation of Haag duality is basically a classical phenomenon. It is well known that commutation properties of free quantum fields correspond to "symplectic orthogonality" of their corresponding wave functions in

$i \operatorname{Im}(f, g)=[A(f), A(g)], \quad f, g$ real test functions.

Hence the quantum A-B effect passes to its classical counterpart; the correct classic vector potential is simply the expec-

\footnotetext{
8 In most articles on the A-B effect the reader is assured that they are unfounded, but they certainly play a role in its popular appeal.
} 
tation value of its quantum counterpart in a suitable coherent state. The Stokes theorem does not contain information as regards the physical localization properties of vector potentials.

The main reason for calling the reader's attention to these facts (well known among experts) is that the unphysical aspects of the quantum gauge formalism are not limited to problems of positivity (unitarity) but they also affect the foundational causal localization principles. The correct localized charge-carrying operators are obtained by smearing directional extended Wightman fields $\Psi(x, e)$ with compactly supported test functions in $x$ and $e$.

The Hilbert space description of massless vector potentials is traditionally presented in the form of Coulomb (or radiation) gauge. Being the unique Hilbert space potential which is rotation invariant in the $t=0$ hyperplane, it is not surprising that it is obtained from integrating the string-local potential over all string directions $e$ in the $t=0$ hyperplane. The lack of covariance and locality prevents its application in renormalized perturbation theory, but it does not impede its use in quantum mechanics.

The phenomenon of breakdown of Haag duality is a general property of all zero mass higher spin fields. For $s=2$ there are two string-local candidates which can be viewed as the analogs of the string-local $A_{\mu}$ namely the string-local $g_{\mu \nu}(x, e)(8)$ or the string-local 3-tensor $A_{\mu \nu \kappa}(x, e)$ which results from a line integration of the field strength $R_{\mu \nu \kappa \lambda}(9)$. The latter plays the analog role to that of the vector potential for $s=1$ in the verification of the breakdown of Haag duality.

It is an interesting question whether in the Platonic world of Haag duality violation there exists a relation between the multiple connectivity (the genus) of the spacetime localization region and the spin of the string-local zero mass potential. It also would be interesting to understand in what sense the construction of covariant string-local potentials can be viewed as a special case of recent constructions in [25].

The important role of positivity for infrared aspects of the QED Hilbert space can be seen by looking at simpler infrared problems in two-dimensional models. The simplest such model is the derivative coupling $\bar{\psi} \gamma_{\mu} \psi \partial^{\mu} \varphi$ of a $d=$ $1+1$ fermion to the derivative of a $m>0$ scalar massive field $\varphi[26]$. It has a solution in terms of a formal exponential expression $^{9}$

$$
\begin{aligned}
& \psi(x)=e^{i g \varphi} \psi_{0}(x), \quad\left\langle e^{i g \varphi(x)} e^{-i g \varphi(y)}\right\rangle=\exp ^{2} i \Delta^{+}(x-y) \\
& \phi:=\lim _{m \rightarrow 0} m^{g^{2}} e^{i g \varphi} \\
& \exp g^{2} i \Delta^{+}(\xi)=F\left(\xi^{2} m^{2}\right) \stackrel{m \rightarrow 0}{\rightarrow}\left(\xi^{2} m^{2}\right)^{-g^{2}} \\
& \quad\left\langle\phi \phi^{*}\right\rangle \neq 0, \quad\langle\phi \phi\rangle=0 .
\end{aligned}
$$

\footnotetext{
9 For reasons of brevity we omit the Wick-ordering in field products at the same point.
}

Although $\varphi$ itself has no positivity-preserving massless limit, the two-point correlation functions of its exponential field $\phi$ remain finite (after rescaling with a $g$-dependent mass factor) and are consistent with conservation of the " $\phi$-charge". In fact the combinatorial structure of the n-point function of $\phi$ in terms of an exponential $i \Delta^{+}\left(x_{i}-x_{k}\right)$ contraction reveals that $\phi$ is a " $g$-charge" conserving field in the Hilbert space which the Wightman reconstruction theorem associates with the limiting $\phi$ vacuum expectation values [2].

There are two aspects of this construction which are worth mentioning. Whereas in the massive case the Hilbert space of the full model is a tensor product of Wigner-Fock $\varphi$-particle space with a $\psi_{0}$ particle space this structure gets lost in the massless limit since the $g$-charge creating the $\phi$ field is not a member of the $\varphi$-Hilbert space rather $\phi$ creates charge sectors in which the charge-neutral $\partial \varphi$ (the limiting $\varphi$ does not exist) acts. Since $\phi$ always appears together with $\psi_{0}$, the Hilbert space is actually a subspace of the tensor product of the $\psi_{0}$ with the $\phi$-space, i.e. the $g$-charge of $\phi$ coalesces with the global $\psi_{0}$ charge. This in turn leads to a kind of kinematical infraparticle structure, which manifests itself in the absence of the mass-shell delta function; the representation of the Poincaré group in the Hilbert space created by the application of $\psi^{\prime} s$ to the vacuum contains no discrete one particle state, but instead of a mass-shell delta function one finds a weaker threshold cut-like singularity structure.

Suppose we ignore the $m \rightarrow 0$ limiting structure of an exponential and define a free $\varphi_{0}$ logarithmic two-point function

$\left\langle\varphi_{0}(x) \varphi_{0}(y)\right\rangle=\log \mu^{2}(x-y)^{2}$.

In this case $\varphi_{0}$ violates positivity i.e. the correlation functions define a linear indefinite metric space. In this case there is no charge superselection structure. The situation resembles that of the use of the Krein space point-local vector potential except that gauge theory restores perturbative positivity for gauge-invariant operators. But a structural understanding of problems behind infrared divergencies of $\mathrm{pl}$ charge-carrying operators within GT is not possible. In the SLFT setting the singular pl siblings $\psi(x)$ disappear in the massless limit and the Maxwell charge-carrying fields exist only as sl fields $\psi(x, e)$. At this point the pl gauge-dependent matter fields $\psi^{K}$ looses its singular pl Hilbert space counterpart $\psi^{P}$.

The understanding of long-range properties of electric charges and the infraparticle aspect in QED pose additional demanding dynamic challenges which go far beyond the kinematical observations on two-dimensional models. There remains, however, a formal analogy with properties one expects in the sl Hilbert space formulations. In order to highlight these analogies it is helpful to reformulate the previous observation by viewing the massless $\phi$-fields as limits of space-like line integrals. With $j_{\mu}=\partial_{\mu} \varphi$ we may define the charge-carrying field $\phi$ directly in the massless model, 
$e^{-i g \varphi}=\lim _{\Lambda \rightarrow \infty} e^{i g \int_{0}^{\Lambda} \mathrm{d} \lambda j_{\mu}(x+\lambda e) e^{\mu}}, \quad e^{2}=-1$.

This suggests the following analogy $\left(\tilde{j}^{\mu}=\varepsilon^{\mu \nu} j_{\nu}\right)$ :

$$
\begin{aligned}
\exp i g \varphi(x, e) & \sim \exp i g \Phi\left(x, e, e^{\prime}\right):=\Psi \\
\text { with } \varphi(x, e) & =\int_{0}^{\infty} j_{\mu}(x+\lambda e) e^{\mu} \mathrm{d} \lambda \text { and } \Phi\left(x, e, e^{\prime}\right) \\
& =\int_{0}^{\infty} A_{\mu}\left(x+\lambda e^{\prime}, e\right) e^{\prime \mu} \mathrm{d} \lambda .
\end{aligned}
$$

In both cases the fields are logarithmically infrared divergent in the massless limit, whereas the exponential operators (defined as above by a Wightman reconstruction from the massless limit of vacuum expectation values) remain finite.

In analogy to the $\phi$ charge we would like to view a state created by $\Psi$ as carrying a Gauss charge i.e.

$$
\begin{aligned}
Q|\Psi\rangle & :=\int \mathrm{d}^{3} x \vec{\nabla} \vec{E}|\Psi\rangle=\lim _{S \rightarrow \infty} \oint_{S} \vec{E} \mathrm{~d} \vec{S}|\Psi\rangle \\
& =\lim _{S \rightarrow \infty} \oint_{S}[\vec{E}, \Psi] \mathrm{d} \vec{S}|0\rangle \neq 0 ?
\end{aligned}
$$

Clearly such a superselected state cannot be compactly localized. Using exponential line integrals over point-local gauge potentials fails, since indefinite metric is not compatible with charges superselection rules, whereas the above ansatz has a better chance. In analogy to the 2-dimensional model the full one-electron state should be of the form $\psi_{0}(x) \Psi(x . \infty)|0\rangle$ where $\psi_{0}$ is a global charge-carrying free matter field and $\Psi$ an infrared photon dressing factor.

One of the few rigorous results in QED is a theorem that the Lorentz symmetry is spontaneously broken in sectors of nontrivial electric charge [27]. This certainly does not happen in interactions with massive vector mesons. The heuristic picture is that the strings of charged particles are the centers of regions of noncompact infrared photon clouds. This is consistent with the established fact that such photon clouds leads to continuously many directional superselection sectors within a fixed charged sector [22]. Whether the above $\Psi$-states have this property can be checked by studying the energy-momentum density in such states.

Returning to the question of the structure of the Hilbert space one may summarize the present situation as follows. Independent of the pl or sl field localization the Hilbert space of asymptotically complete theories with a mass gap is a Wigner-Fock particle space where the particles are related to the interacting fields by LSZ scattering theory. This is a very clear conceptual situation. In massless limits involving sl $s \geq 1$ potentials this picture breaks down; in such cases the structure of the Hilbert space is expected to involve massless limits of nonpolynomial (in the case of QED exponential) string-local new sector creating composites involving massless potentials. In problems with a mass gap, one expects the
Wigner-Fock space provided by scattering theory to be complete ("asymptotic completeness"). In a perturbative setting this is part of the formalism.

The reconstruction of a Hilbert space from massless limits of correlation functions in terms of suitable limits of free fields is expected to require the use of exponential non-local limits of free fields (28) which create inequivalent representation spaces. But even in the case the above proposal to describe the Hilbert space of QED turns out to be correct, there remains the problem how these inequivalent representations generating descriptions are spacetime-related to the interacting fields. Such constructions are expected to lead to a better understanding of the momentum space recipes [28,29] in terms of spacetime concepts of collision theory.

Morchio and Strocchi addressed the infrared problem within the gauge theoretic setting by constructing positivityobeying topology on the Krein space formalism [6]. We believe that the understanding of such long-distance problem will be simpler and more natural in a description where these string-local objects are already part of the perturbative setting and the topology of the Hilbert space is already the correct one.

A intriguing proposal can be found in a recent article by Buchholz and Roberts [30]. These authors observe that a restriction of the Minkowski space to a forward light cone $V_{+}$would still permit a complete description of QFTs with a mass gap, but its irreducibility would be lost in the presence of photons. In the context of string-local fields this situation suggests to use fields localized on space-like hyperbolic curves which stay inside $V_{+}$and only touch the surface of the light cone at light-like infinity. Such a situation could lead to a more natural way to implement infrared cut-offs. What is missing is a perturbative realization of this idea; but the increasing perturbative experience with fields localized on space-like lines suggests that such an extension may be possible.

\section{Differential-geometric control of directional fluctuations}

Whereas setting up first order string-local interactions in the form of $L, V_{\mu}$ pairs within the power-counting restriction is basically a kinematic problem involving free fields, the situation changes when it comes to the construction of the higher order S-matrix. The reason is that the singular nature of time ordering does not permit one to take the divergence $\partial^{\mu} T\left(. . \partial_{\mu} \varphi \ldots\right)$ directly through the time-ordering to the affected operator. The differential relation ${ }^{10}$

\footnotetext{
10 Unpublished notes by Jens Mund (Vienna 2012).
} 


$$
\begin{aligned}
& d\left(T L L^{\prime}-\partial^{\mu} T V_{\mu} L^{\prime}-\partial^{\prime \mu} T L V_{\mu}^{\prime}+\partial^{\mu} \partial^{\prime \mu} T V_{\mu} V_{\mu^{\prime}}^{\prime}\right)=0, \\
& \quad d=d_{e}+d_{e^{\prime}}, \\
& \left.T L L^{\prime}\right|^{P}:=T L L^{\prime}-\partial^{\mu} T V_{\mu} L^{\prime}-\partial^{\prime \mu} T L V_{\mu}^{\prime}+\partial^{\mu} \partial^{\prime \mu} T V_{\mu} V_{\mu^{\prime}}^{\prime},
\end{aligned}
$$

which secures the $e$-independence of the second order Smatrix in the adiabatic limit (formally the integral over Minkowski spacetime), ${ }^{11}$ would be a trivial consequence of (16) if it were not for singularities in $T$-products from coalescent points and crossing of strings.

In terms of differential forms in the de Sitter space of directions the individual contributions to the right hand side are zero forms and their sum is an exact form. We remind the reader that in the presence of a mass gap there are no boundary terms at infinity, so that in analogy to (17) the divergence terms do not contribute to the second order S-matrix, hence

$\left.S^{(2)} \sim \iint T L L^{\prime}\right|^{P}=\iint T L L^{\prime}$

It turns out that such "normalization" problems as posed by (30) can be solved by using the freedom in defining timeordered products. Starting from a "kinematic" time ordering $T_{0}$, one computes the anomaly $A$ as the singular part of the terms containing derivatives

$$
-A=s \cdot p \cdot\left(-\partial^{\mu} T_{0} V_{\mu} L^{\prime}-\partial^{\prime \mu} T_{0} L V_{\mu}^{\prime}+\partial^{\mu} \partial^{\prime \nu} T_{0} V_{\mu} V_{v}^{\prime}\right) \text {. }
$$

Since in the following we will be interested in the S-matrix, we only need the contribution from the 1-contraction (the tree component) $\left.A\right|_{1-\text { contr. }}$; for notational economy we will omit the subscript, so in the following relations $A$ stands for the tree component of the anomaly.

The kinematic $T_{0}$ is defined by taking all derivatives outside e.g.

$$
\begin{aligned}
\left\langle T_{0} \partial \varphi \partial^{\prime} \varphi^{* \prime}\right\rangle & :=\partial \partial^{\prime}\left\langle T_{0} \varphi \varphi^{* \prime}\right\rangle \\
\partial^{\mu}\left\langle T_{0} \partial_{\mu} \varphi \varphi^{* \prime}\right\rangle & =-i \delta\left(x-x^{\prime}\right)-m^{2}\left\langle T_{0} \varphi \varphi^{* \prime}\right\rangle .
\end{aligned}
$$

If the anomaly is of the form

$A=-N+\partial^{\mu} N_{\mu}+\partial^{\mu} \partial^{\nu} N_{\mu \nu}$

where the $N^{\prime} s$ contain $\delta\left(x-x^{\prime}\right)$ functions, they can be absorbed as renormalization terms in the time-ordered operator products (30). They describe the delta function terms which violate Eq. (30) if one uses the kinematical $T_{0}$; hence the anomaly terms reveal in what way the time-ordered products at coalescent points (or more general at string intersections) in (30) have to be defined.

For the calculation of the second order S-matrix one only needs to compute the $N$. Note that $N$ terms are similar to

\footnotetext{
11 In massive theories boundary terms at infinity vanish. Without $\mathrm{x}, \mathrm{x}$ ' integration the expression in the bracket can be used as a definition of a second order point-local interaction density.
}

the counterterms well known from the renormalization formalism for point-local interactions. But there is a significant conceptual difference. Whereas the renormalization counterterms in point-local renormalization come with new coupling parameters, the contact terms originating from anomalies are uniquely determined in terms of the basic first order couplings and the masses of the free fields in terms of which the first order interaction density is defined. Such anomaly terms will be referred to as induced interactions. They originate from the implementation of the e-independence of the $S$-matrix and hence they have no analog in $s<1$ point-local interactions.

The calculation of the S-matrix requires only the calculation of $N$. In that case it is more convenient to use a weaker formulation

$d L-\partial^{\mu} Q_{\mu}=0, Q_{\mu}:=d_{e} V_{\mu}$

$d T L L^{\prime}-\partial^{\mu} T Q_{\mu} L^{\prime}-\partial^{\prime \mu} T L Q_{\mu}^{\prime}=0$.

This " $Q$-formulation" is closely related to the implementation of the gauge-invariant S-matrix in $\mathfrak{s} S=0$ in the CGI BRST setting.

The $V$-formalism, which leads to the definition of pointlocal interactions densities (30), turns out to be indispensable for the construction of interacting string-local fields. It permits to define higher order point-local interaction densities in terms of the renormalizable string-local formalism. Together with the formal relation between point- and string-local matter fields (6) it can be used for the perturbative construction of renormalized correlation functions of string-local fields. The point-local higher order interaction densities (30) play the role of the $e$-independence of string-local field correlations from the $e^{\prime} s$ of internal propagators. This construction of interacting string-local fields has been initiated by Jens Mund and will be the subject of a forthcoming publication [21].

Assuming that these string-local fields remain renormalizable (Wightman fields in $x$ ) in every order, the results of Jaffe [7] on the singular nature of exponentials of scalar free fields suggest that may exist as a singular (not Wightmanlocalizable) field in a well-defined sl Wightman theory. As previously mentioned the similarity of the relation between the pl Proca potential and its sl counterpart with an operator gauge transformation suggests that the renormalizable sl matter field is exponentially related to a singular pl field $\psi^{P}(x)=\psi(x, e) e^{-i g \phi}$ in which the escort field plays the role of a gauge parameter. Whether this definition of a singular $\mathrm{pl}$ field within a renormalizable sl model permits a mathematical control as a singular field in the sense of Jaffe, which depends only on the same finite number of physical coupling parameters as the sl field, remains an interesting open question.

This indicates that the nonrenormalizability of certain $\mathrm{pl}$ models is the result of forcing the sl localization of $s \geq 1$ 
fields into a pl setting at the price of unbounded $d_{s d}$ and (even worse) a predictability destroying unbounded increase of coupling parameters. If the pl fields defined within the sl setting turn out to have a mathematical existence in the sense of Jaffe then their unbounded off-shell high energy behavior should permit an on-shell restriction which fulfills the perturbative expected unitarity restriction. In this case one expects that the sl formulation fixes the free counterterm parameters of the nonrenormalizable pl description in such a way that its on-shell restriction (through high energy cancellations) agrees with that of the sl description.

Another important new concept which has no analog in $s<1$ pl interactions is the above-mentioned appearance of induced terms. In the following this will be illustrated in terms of sl second order calculations in three different models.

The first such model is scalar massive QED (13). In the case the anomaly contribution arises from the divergence acting on the two-point function involving a $\partial_{\mu} \varphi$. The result for the induced contact contribution is the expected second order quadratic in $A_{\mu}$ contribution

$g^{2}: \varphi^{*}(x) A_{\mu}(x, e) \delta\left(x-x^{\prime}\right) \varphi\left(x^{\prime}\right) A^{\mu}\left(x, e^{\prime}\right):+\left(e \leftrightarrow e^{\prime}\right)$,

which may be absorbed into a change of the $T_{0} \rightarrow T$ product of the $\partial \varphi \partial^{\prime} \varphi^{* \prime}$ contraction contributing

$$
\begin{aligned}
& \left\langle T_{0} \partial_{\mu} \varphi^{*} \partial_{\nu} \varphi\right\rangle \rightarrow\left\langle T \partial_{\mu} \varphi^{*} \partial_{\nu} \varphi\right\rangle \\
& \quad=\left\langle T_{0} \partial_{\mu} \varphi^{*} \partial_{\nu} \varphi\right\rangle+c g_{\mu \nu} \delta\left(x-x^{\prime}\right) .
\end{aligned}
$$

For more details we refer to [18].

This is similar to gauge theory where the induction of the second order term quadratic in $A$ results from BRST gauge invariance of the S-matrix ${ }^{12} \mathfrak{s} S=0$. In SLFT the independence of the $\mathrm{S}$-matrix from string directions is a natural physical consequence of large-time scattering theory [14] for string-local fields in the presence of a mass gap. In a perturbative setting, which is based on the construction of the S-matrix in terms of the adiabatic limit of Bogoliubov's formal time-ordered products of interaction densities, it has to be imposed (30).

There is a fine point which turns out to be of significant conceptual importance. The directional fluctuations only disappear after adding up all contributions to a particular scattering amplitude in the same perturbative order. For the case at hand the singularity at $e=e^{\prime}$ in the time-ordered propagator (which enters the second order tree contribution to

\footnotetext{
12 In the naive formulation the quadratic term arises from the replacement of $\partial \rightarrow \partial-i g A$ in order to preserve gauge invariance of the classical Lagrangian.
}

scattering) corresponding to the two-point function (5),

$$
\begin{aligned}
\frac{1}{p^{2}-m^{2}-i \varepsilon}( & -g_{\mu \mu^{\prime}}-\frac{p_{\mu} p_{\mu^{\prime}}\left(e \cdot e^{\prime}\right)}{(p \cdot e-i \varepsilon)\left(p \cdot e^{\prime}+i \varepsilon\right)} \\
& \left.+\frac{p_{\mu} e_{\mu^{\prime}}}{(p \cdot e-i \varepsilon)}+\frac{p_{\mu} e_{\mu^{\prime}}^{\prime}}{\left(p \cdot e^{\prime}+i \varepsilon\right)}\right)
\end{aligned}
$$

is ill defined since the two fluctuating directions enter the two-point distribution with a different $\varepsilon$-prescription ${ }^{13}$ from the Fourier transformation of the two Heaviside functions. But in the use of this propagator for the second order on-shell scattering amplitude (the scalar analog of Møller and Bhabha scattering) this problem disappears. This is similar to the verification of on-shell gauge invariance in the second order tree approximation, except that $e, e^{\prime}$ are not global gauge parameters in a Krein space gauge theory but rather space-like directions of independently fluctuating string-local vector potentials acting in a Hilbert space.

Whereas in the Krein space gauge setting an individual gauge-dependent perturbative contribution to a scattering process exists, the independently fluctuating $e^{\prime} s$ become only "mute" after adding up sufficiently many perturbative on-shell contributions in a fixed order of $S$ (akin to on-shell gauge invariance). Any attempt to interpret the independently fluctuating $e^{\prime} s$ as gauge parameters by equating them in contributions from different fields causes the renormalization resistant infinities of the abandoned axial gauge formalism to return with full vengeance.

It is important to notice that the second order $A \cdot A \varphi^{*} \varphi$ contribution is induced by the principles of QFT (causal localization in Hilbert space), there is no reference to the substitution rule $\partial \rightarrow D=\partial-i g A$ of classical gauge connections. The SLFT setting of QFT does not refer to substitution rules or the theory of fiber bundles, but rather explains the appearance of such terms as an inherent property of a QFT in the presence of positivity-maintaining sl vector mesons.

This is even more remarkable in models of self-interacting vector mesons in which the Lie-algebra structure, unlike classical gauge theory, bears no relation to an imposed symmetry, but rather results from only implementing the spacetime causality principles. That the classical causality principles of Faraday, Maxwell, and Einstein become that powerful in the context of Hilbert space positivity of interacting higher spin $s \geq 1$ quantum matter is truly surprising.

An even richer second order induction, which has no counterpart in classical field theory, is expected to, occurs in the $g A \cdot A H$ coupling of massive vector mesons to a Hermitian matter field $H$ (14). One observes an analogy to the requirement of BRST invariance of the S-matrix up to a third order tree approximation [9]. This did not only generate the expected second order $A \cdot A H^{2}$ contributions which, as the

\footnotetext{
13 This is the reason why the axial gauge interpretation failed.
} 
induced $A \cdot A|\varphi|^{2}$ contribution of scalar QED can be absorbed in a redefinition of the time-ordered product in analogy to (37), but also induces Mexican hat-like self-interactions of $H$. Thus, one also expects the $e$-independence of the S-matrix to induce such self-interactions.

This second order induction was first seen in the CGI setting of the BRST gauge formalism where it results from the implementation of the BRST invariance $\mathfrak{s} S=0$ of the Smatrix [9]. The reason why its was overlooked (and confounded with a SSB) is presumably that in the standard timeordered formalism it is difficult to separate off-shell properties from those which only hold on-shell and as a result the on-shell induction is easily confused with an off-shell SSB Higgs mechanism. The direct implementation of BRST invariance in an operator setting, in which the S-matrix is seen as a result of the spacetime adiabatic limit rather than of a momentum space mass-shell restriction, helps to avoid such confusions.

The BRST gauge setting introduced many new algebraic objects into GT but it did not completely cut the umbilical cord with classical gauge theory. This is the origin of its problem with positivity (unitarity), a property which has no natural place in classical field theory. It remains a patchwork of important physical islands (the subalgebra of local observables and the S-matrix) which are formally interconnected by unphysical gauge-dependent fields, in which negative metric degrees of freedom and ghost fields remain present. In the words of one of its main protagonists (Raymond Stora) "GT is the amazingly successful placeholder of an unknown QFT". SLFT is an attempt to substitute this placeholder by a full Wightman QFT.

The lack of positivity of GT affects also the causal localization principles of QFT; the topological $e$-dependence of free Wilson loops, as presented in the previous section, is the simplest illustration. The causality violations of gaugedependent fields are more serious. Gauge symmetry and gauge invariance are not concepts which can be directly related to or derived from the principles of QFT. The physical reason why gauge-dependent fields are unphysical is that in addition to not accounting correctly for the quantum probability they also do not present the correct causal localization. The noncompact localization of charges in QED [8] can only be accounted for in terms of test-function-smeared sl chargecarrying fields.

Perhaps the most important new message coming from the positivity maintaining sl setting is the appearance of the degrees of freedom maintaining escort fields. For $s=1$ they are new scalar covariant fields which do not carry any degrees of freedom of their own and which in the absence of interactions depend only on the Wigner momentum space $s=1$ creation/annihilation operators. Their existence and purpose is overlooked in standard quantization settings because there are no pl escorts.
These escorts become indispensable dynamic objects in the presence of interaction; they constitute the physical counterpart of the unphysical additional negative metric and ghost degree of freedoms. These escorts (and not the Hermitian $H$ fields) are the abelian Higgs model, are the objects which comply with the analogy to the bosonic Cooper pair which appears in the famous paper by Higgs [31]. They are the objects which cannot be avoided in descriptions of how massive Proca fields are related to massless vector potentials in a positivity-maintaining way.

At this point a surprised reader may ask why the presence of these objects was not noticed during the almost seven decades since the discovery of QED. The simple answer is: there never has been a positivity-obeying QFT of $s \geq 1$ models; higher spin interactions were always described in a Krein space gauge setting and $s \geq 1$ Lagrangian quantization does not lead to anything else but $\mathrm{pl}$ indefinite metric fields requiring a gauge-theoretic setting. Wigner's unitary representation theory has existed since 1939 [32] and years later Weinberg developed the intertwiner formalism which converts Wigner's wave functions into pl covariant fields [33]; but when it came to models involving vector mesons (QED and the nonabelian vector mesons of the Standard Model) he used the gauge theory of Lagrangian quantization.

The Hilbert space setting required a resolution of the clash between positivity and localization. This became possible after the intertwiners for sl localization were constructed [1] within the setting of modular localization in Wigner representation spaces [15].

The interactions of abelian massive vector mesons with $H$ fields are subject to the same principles as couplings to complex matter (massive spinor or scalar QED). The difference is that Hermitian matter is not subject to global charge conservation; this accounts for the fact that the $L, V_{\mu}$ pair condition requires the presence of the escort field already in the first order interaction density $L$ and leads to the richer induction, which includes the $H$ and $\phi$ self-interactions. Last but not least, the Higgs interaction vanishes (together with the disappearance of the escort) in the limit of massless vector mesons, i.e. Higgs matter disappears in the massless Maxwell limit.

These differences between interactions of complex and real matter with massive vector mesons may explain why the Higgs model escaped the systematic study of renormalizable interactions and why it was discovered in the roundabout way through the application of a SSB prescription to the two-parametric gauge theory of scalar QED modified by a tachyonic mass term (the Mexican hat potential). It may also account for the strange fact that the discovery (different from all other discoveries which led to Nobel prizes) was made independently in at least three independent papers using almost identical calculations including the well-known 
metaphor ${ }^{14}$ "fattening of massless vector mesons by incorporating massless Goldstone degrees of freedom".

The important role of the escort fields in the relation between massless and massive vector mesons permits one to see in more clarity why the presence of $H$ fields in models containing self-interacting massive vector mesons is of fundamental importance. The relevant observation is that, although there is a first order interaction density of selfinteracting vector mesons which passes the sl preserving $L, V_{\mu}$ pair condition, there is a $\mathrm{PCB}$ violating induced second order $d_{s d}=5$ contribution. Since the improvement of PCB through weakening of localization was already used in the construction of the first order $L, V_{\mu}$ pair, the only remaining PCB improving resource is to extend the first order interaction density by a coupling to an additional field and hope for a compensation of a newly created $d_{s d}=5$ term with that induced from the $s=1$ self-interaction.

The guiding analogy behind such a construction is the picture of improved short-distance behavior in supersymmetric multiplets as a consequence of compensations between different spin components within the same multiplet. Whereas in the case of supersymmetry this is a consequence of the extended symmetry, the existence of a compensatory field for the case at hand is its raison d'être. The looked-for compensating field cannot have a spin $s \geq 1$ (higher spin would worsen the second order $d_{s d}$ ) and it should maintain the Hermiticity of the vector mesons, hence it must be a $H$-field. The SSB Higgs mechanism hides this compensatory preservation of the second order PCB.

The absence of a SSB can also be seen by looking directly at the resulting model since the charge of a conserved current of an abelian massive vector meson (the identically conserved Maxwell current) is always screened $(Q=0)$ and never spontaneously broken $(Q=\infty)$ whereas in the presence of self-interacting vector mesons there exists no conserved current.

There remains the question why two such quite different ideas as the Higgs mechanism and that used in the present work lead to the same interaction density including the $H$ self-interactions. The answer is quite simple and is rooted in the nature of QFT. In contrast to quantum mechanics, a specific model of QFT is already nailed down by prescribing the first order field content (including the masses of the free fields and internal symmetries). One cannot construct different QFT with the same field content. For the case at hand this implies that any renormalized interaction between a massive vector potential and a Hermitian field must be equal to the Higgs model. Its physical properties are intrinsic and do not depend on the ideas in terms of which the calculating

\footnotetext{
14 Metaphors occasionally played an important role in discoveries within highly speculative areas of research; they served as temporary placeholders for a future foundational understanding.
}

physicist obtained the model. Screened Maxwell charges of abelian massive vector mesons are very different from divergent SSB charges.

As is well known from renormalizable $s<1 \mathrm{pl}$ interactions, the counterterm formalism generates all possible contributions to the renormalizable interactions between the fields of the given field content even if one started with only some of them. This picture is strengthened in the presence of sl $s \geq 1$ fields for which the $L, V_{\mu}$ condition for the preservation of sl localization leads to the phenomenon of the parameter-preserving "induction" 15 and the preservation of higher order PCB through compensatory extensions of the original field content.

Presently one does not know whether these new ideas permit $s>1$ interactions. The reason is that even after having been able to satisfy the $L, V_{\mu}$ pair condition there may be second order induced $d_{s d}>4$ contributions. As in the case of self-interacting massive vector mesons the only remaining remedy is the cancellation of such terms by enlargement of the first order field content. As long as this can be achieved, the model exists in the sense of perturbative QFT.

A family of fields which do not pass the $L, V$ pair requirement is the class of infinite spin Wigner representations. In this case the presence in $L$ of an sl field from that class violates this requirement and therefore cannot interact within the rules of QFT with any other field. Such fields are called inert or nonreactive. They only exist as free fields but as positive energy representations they may interact with the classical gravitational field through their energy-momentum tensor [11].

All higher spin fields with $s<\infty$ have conserved energymomentum tensors which in the massless case are necessarily string-local. It is an interesting theoretical question whether there is a "critical" finite spin $s_{\text {crit }}>1$ above which fields are inert. The study of the pair condition for higher spin is expected to reveal the answer.

It is not unreasonable to believe that the sl extended perturbation theory marks the true borders within which models of QFT exist. This does not necessarily contradict the lack of converge (in certain models known and generally expected) of perturbation theory. There are many possible explanations why renormalized perturbation theory cannot be used for establishing the mathematical existence of a model of QFT.

The localized fields may be too singular for their use in existence proofs of models of QFT. The idea that the avoidance of singular pl or sl fields may be important for existence proofs of models of QFT is corroborated by the "top-down" construction of certain two-dimensional integrable models in which one starts with their known S-matrix which contains information as regards the algebraic structure of the wedge-

\footnotetext{
15 The difference from the generation of counterterms is that induced contributions do not enlarge the number of coupling parameters.
} 
localized algebras. By showing the existence of nontrivial intersections corresponding to compact localized algebras one arrives at the construction of a system of local algebras which fulfills all the properties of an algebraic QFT [12]. The question of whether it contains generating $\mathrm{pl}$ or sl fields remains open.

\section{Gauge theory and local quantum physics}

Despite significant conceptual differences between GT and SLFT there are also formal analogies. A comparison between these two ways of describing QFT of $s=1$ fields leads to a better understanding of their mutual relation. In the absence of interactions the differences only affect a subtle point in the interpretation of causality in gauge-invariant Wilson loops (Sect. 3), but they increase in the presence of interactions. The construction of the globally gauge-invariant S-matrix is a good illustration of the conceptual differences between the two settings.

The CGI BRST operator formalism handles this problem in the following way. Instead of (4) one writes

$A_{\mu}^{K}(x)-\partial_{\mu} \phi^{K}=: A_{\mu}^{P, K}(x)$

where $K$ refers to Krein space, $A_{\mu}^{K}$ is the massive vector potential in the Feynman gauge, $\phi^{K}$ is a massive free scalar field with the opposite sign in its two-point function (the auxiliary "Stückelberg field"), and $A_{\mu}^{P, K}$ is a substitute for the Proca potential which does not explicitly appear in the Krein Fock space but plays the role of emulating Wigner particle states in expectation values or matrix-elements of gauge invariant operators between such states to space.

As noticed by Mund (private communication), the Krein space candidate of a Proca field has indeed the same twopoint function as its Hilbert space analog; but being an object in a larger Krein space (the tensor product of two Krein space it is at only a cohomolgical emulation of a physical Wigner operator. Such emulations of Wigner states searched for but overlooked in [34].

Whereas $\phi^{K}$ adds unphysical degrees of freedom and remains in the massless limit, the SLFT sl escort $\phi$ results from a rearrangement of existing physical degrees of freedom $^{16}$ and plays no role in the properties of the massless limit.

Using the ghost rules (10) one defines a $L^{K}, Q_{\mu}^{K}$ pair which fulfills [9]

\footnotetext{
16 Remember the analogy to Cooper pairs from rearrangements of condensed matter degrees of freedom which causes the short range nature of vector potentials in the superconducting phase.
}

$\mathfrak{s} L^{K}-\partial^{\mu} Q_{\mu}^{K}=0, \quad$ hence $\mathfrak{s} S^{(1)}=0$, $\mathfrak{s} T L^{K} L^{K \prime}-\partial^{\mu} T Q_{\mu}^{K} L^{\prime K}-\partial^{\prime \mu} T L^{K} Q_{\mu}^{\prime K}=0$, hence $\mathfrak{s} S^{(2)}=0$.

In analogy to the $e$-independence (35), but with the significant conceptual difference that, whereas $d$ acts on the individual spacetime string directions, the globally acting BRST nilpotent $\mathfrak{s}$ has no physical interpretation of its own, but it only serves to extract a physical S-matrix from an unphysical point-local Krein space description.

The formal analogy of the SLFT $Q$ formalism (35) with (39) stands in contrast to the quite different behavior of the two $Q^{\prime} s$ in the massless limit which only exists for $Q^{K}$. The indefinite metric has its strongest unphysical manifestation in the GT of quantum electrodynamics since a physical $\mathrm{pl}$ electron field coupled to massless vector mesons does not exist, not even as a singular field in the sense of (6), since the escort field $\phi$ (in contrast $\phi^{K}$ ) diverges as $m^{-1}$ for $m \rightarrow 0$.

Whereas the gauge formalism provides no warning concerning fundamental conceptual changes, the positivity respecting SLFT signals these changes in the relation between fields and particles (the breakdown of scattering theory and the Wigner-Fock particle structure of the Hilbert space) through the breakdown of its perturbative formalism (the $m^{-1}$ divergencies in the $V_{\mu}, Q_{\mu}$ ).

The important message from the SLFT perturbation theory is to avoid the direct construction of the operators and the Hilbert space in the presence of massless vector mesons and instead obtain the massless theory through the massless limit of the massive vacuum expectation values (SLFT Wightman functions). This extends the almost trivial free field observation that (despite the significant difference of the Wigner operators of massive spin and massless helicity representations) the $s l$ two point correlation functions of former pass to those of the latter. The differences on the level of operators and Hilbert space would reappear if one uses the correlation functions in a Wightman operator reconstruction.

Since the S-matrix is gauge invariant it is plausible that its perturbative logarithmically infrared divergent QED limit can be used to extract prescriptions for gauge-invariant photon-inclusive cross sections (Bloch-Nordsiek [28] and its relativistic YFS refinement [29]). The same problem exists in SLFT, but being a full QFT with a physical localization in Hilbert space, it is a better starting point for a future spacetime understanding of "infraparticles" and their collision theory. One conjecture is that the long-ranged string localization of charge-carrying fields "dissolves" the mass shell and converts it into a weaker cut-like singularity at the electron mass; this would lead to a vanishing large-time (LSZ) limit for a collision of charges particles with a finite number of outgoing photons and explain the formal necessity to sum over infinitely many infrared photons. 
Another problem for which the SLFT setting is expected to play a pivotal role is the issue of confinement. Here the important observation is that all massless model of GT in all covariant gauges are free of off-shell infrared problems; for nonabelian GT this has been shown in the work of Hollands [35].

This is expected to change in SLFT since the independent directional fluctuations in the presence of sl fields lead to stronger long-distance fluctuation. If this leads to off-shell logarithmic divergencies in correlations containing in addition to pl hadronic composite fields also sl gluon and quark fields, this would be a very strong indication that the resummation of logarithmic mass divergencies and the subsequent $m \rightarrow 0$ limit in fact leads to a vanishing of those correlations functions, so that only pure hadronic and gluonium correlations remain. The quarks and gluons disappear, but their traces are imprinted on the observed particles.

A good insight into the differences between GT and SLFT requires to understand the conceptual position of the Coulomb/radiation gauge. As a vector potential which lives in Hilbert space it historically precedes sl fields; but due to its lack of covariance it was not suited for the use in renormalized perturbation theory. Since it is the only positivitymaintaining rotational invariant vector potential, it must be identical to the rotational averaged sl potential in which the space-like $e$ is integrated over a unit sphere in a space-like hyperplane (this is not a gauge transformation). Hence both potentials live in the same Hilbert space.

As a positivity-obeying vector potential it is not a member of GT in the sense that this terminology was used in the present paper, where it is reserved for cases in which indefinite metric degrees account for the freedom of gauge transformations. Radiation-like potentials exist for all sl tensor potentials.

There are some puzzling observations about symmetries, which originally came from the CGI perturbative implementation of on-shell gauge invariance $\mathfrak{s} S=0$. Starting with the most general PCB obeying ansatz of an interaction density of self-interacting massive vector mesons,

$L=\sum f_{a b c} F^{a \cdot \mu \nu} A_{\mu}^{K, b} A_{v}^{K, c}+\cdots$

with arbitrary real coupling coefficient $f_{a b c}$ and additional contributions containing $\phi^{K}$ and ghosts, one finds that the onshell gauge invariance of $S$ (39) leads to strong restrictions of the parameters $[9,10]$. In particular, the $f_{a b c}$ have to satisfy the Lie-algebra relation of the adjoint representation. This holds even if the masses are different. ${ }^{17}$

Although the more involved second order calculations in the SLFT setting have not been finished at the time of writing, the formal similarities between the implementation of

\footnotetext{
17 The restrictions from gauge invariance lead to restrictions on the masses but do not require them to be equal.
}

operator gauge invariance (39) and the delocalization preventing $L, Q_{\mu}$ restriction strongly suggest that this property also holds in SLFT. This would realize an old dream of Raymond Stora proving that the Lie-algebra structure of self-interacting vector mesons is a consequence of the foundational causal localization principles of QFT and did not enter through the quantization parallelism to classical gauge theory.

This symmetry is very different from the concept of inner symmetries according to which every symmetric theory can be converted into a less symmetric model (more coupling parameters) by maintaining its field content but allowing the masses of the multiplet to be different.

Inner symmetries are well understood within the Doplicher-Haag-Roberts superselection theory according to which the superselection sectors of an observable algebra have the composition structure of the dual of a compact group [22].

The Lie structure of self-coupled vector mesons on the other hand is different since it continues to exist in the presence of unequal masses and does not permit a weakening toward a less symmetric self-interaction, Hence attempts to explain it as a SSB of an internal symmetry are digressive. This applies in particular to the proposal to the formal interpretation of gauge symmetry as an inner symmetry in a Krein space setting.

This phenomenon does not occur for $s<1$; there all symmetries are conventional inner symmetries. It would be interesting to see whether there exist consistent higher spin models containing $s>1$ self-interacting sl potentials in with the self-couplings come with an intrinsic Lie group structure.

This could explain why theoreticians using methods of algebraic QFT always had problems with gauge symmetries [22] and why a foundational understanding requires new ideas [30]. It would be very interesting to see whether the perturbative results within SLFT can shed new light on these age old problems of local quantum physics.

\section{An outlook}

As stated in the introduction, the principal motivation for writing this paper is to direct attention to the beginnings of a new development in QFT whose aim is to preserve renormalizability within a Hilbert space setting. The present attempt focuses on those conceptual properties which distinguish the new setting from that of gauge theory, but left out important mathematical details, partly because they do not yet exist and also because those which are meanwhile available will be contained in forthcoming work by Jens Mund [20,21].

The main aim of this paper is the presentation of some ideas about an extension of renormalization theory to inter- 
acting string-local vector mesons. Since $d_{s d}=1$ for all sl $s \geq 1$, the mere fulfillment of the $d_{s d}^{\text {int }} \leq 4$ power counting restriction for controlling short distances in terms of sl fields is easy, but there arises a new large-distance problem which has no analog for $s<1$ interactions. The new danger is now that the sl interaction leads to completely delocalized fields in higher orders. To avoid this, the first order sl interaction density $L$ must be part of a so-called $L, V_{\mu}$ pair which turns out to be a rather strong restriction whose implementation and consequences have only been studied for sl vector mesons.

Even after its fulfillment there may be a higher order violation of PCB which requires to extend the model by compensating contributions from couplings with additional lower spin fields. The presently only known illustration of this compensatory mechanism is provided by self-interacting vector mesons in which case the compensating lower spin fields are Hermitian scalar $H$ fields.

The new setting does not invalidate gauge theory, it rather highlights its restricted physical range. GT permits a correct description of the S-matrix of interaction massive vector mesons and local observables, but the physics behind gauge-variant fields remains outside its physical range. This includes the problem of quark confinement and also the spacetime understanding of scattering problems in QED. Within the limitations of gauge invariance, gauge theory is a very successful placeholder of a QFT of $s=1$ particles.

Disregarding structural theorems (TCP, Spin\&Statistics, derivation of large-time scattering, etc.) whose proof requires Hilbert space positivity [2], the BRST perturbative formulation accounts for the perturbative gauge-invariant local observables (field strength, currents) and - which guaranteed the success of GT in the Standard Model —of the perturbative unitary S-matrix. What is missing are the matter fields which relate the world of the causal localization principles of QFT with the measurable world of Wigner particles. The close connection between positivity and causality prevents GT to account correctly for the physical localization properties at large distances (infrared problems).

In order to go beyond gauge theory, the SLFT Hilbert space setting for the S-matrix must be extended to the calculation of string-local correlation functions, a task which goes significantly beyond the construction of the stringindependent S-matrix.

Acknowledgments This work is part of an ongoing project with Jens Mund; its main purpose is to strengthen historical roots and interconnections with other results. I am deeply indebted for his advice and suggestions. For informations about the early history of the standard model I owe thanks to José Gracia-Bondia and Joseph Várilly. Raymond Stora, who sadly passed away on June 20th this year (2015), has been my mentor during the last two years; his last mail (always surface mail) is dated from 30.05.2015.
Open Access This article is distributed under the terms of the Creative Commons Attribution 4.0 International License (http://creativecomm ons.org/licenses/by/4.0/), which permits unrestricted use, distribution, and reproduction in any medium, provided you give appropriate credit to the original author(s) and the source, provide a link to the Creative Commons license, and indicate if changes were made.

Funded by SCOAP ${ }^{3}$.

\section{References}

1. J. Mund, B. Schroer, J. Yngvason, String-localized quantum fields and modular localization. CMP 268, 621 (2006). arXiv:math-ph/0511042

2. R.S. Streater, A.S. Wightman, PCT, Spin and Statistics and All That (Benjamin, New York, 1964)

3. P. Jordan, Zur Quantenelektrodynamik, I. Eichinvariante Operatoren. Zeitschrift für Physik 95, 202 (1935)

4. S. Mandelstam, Quantum electrodynamics without potentials. Ann. Phys. 19, 1 (1962)

5. O. Steinmann, Ann. Phys. 157, 232 (1984)

6. G. Morchio, F. Strocchi, J. Math. Phys. 44, 5569 (2003)

7. A. Jaffe, Phys. Rev. 158, 1454 (1967)

8. D. Buchholz, Commun. Math. Phys. 85, 40 (1982)

9. G. Scharf, Quantum Gauge Theory, A True Ghost Story (Wiley, New York, 2001)

10. M. Duetsch, J.M. Gracia-Bondia, F. Scheck, J.C. Varilly, Quantum gauge models without classical Higgs mechanism. Eur. Phys. J. C 89, 599 (2012). arXiv: 1001.0932

11. B. Schroer, Can the inert matter corresponding to Wigner's infinite spin representations be dark matter? EPJC (submitted)

12. G. Lechner, Algebraic constructive quantum field theory: integrable models and deformation techniques. arXiv:1503.03822

13. S. Weinberg, E. Witten, Limits on massless particles. Phys. Lett. B 96(1-2), 59 (1980)

14. D. Buchholz, K. Fredenhagen, Locality and the structure of particle states. Commun. Math. Phys. 84, 1 (1982)

15. R. Brunetti, D. Guido, R. Longo, Modular localization and Wigner particles. Rev. Math. Phys. 14, 759 (2002)

16. B. Schroer, Modular localization and the holistic structure of causal quantum theory, a historical perspective. SHPMP 49, 109 (2015)

17. J. Mund, in String-Localized Quantum Fields, Modular Localization and Gauge Theories. New Trends in Mathematical Physics, ed. by V. Sidoravicius. Selected Contributions of the XVth Int. Congress on Math. Physics (Springer, Dordrecht, 2009), p. 495

18. B. Schroer, Peculiarities of massive vector mesons and their zero mass limits. Eur. Phys. J. C 75, 365 (2015)

19. A. Aste, G. Scharf, M. Duetsch, J. Phys. A 30, 5785 (1997)

20. J. Mund, E.T. de Oliveira, String-localized free vector and tensor potentials with any spin: I. Bosons (in preparation)

21. J. Mund, String-localized massive vector bosons without ghosts and indefinite metric: the example of massive QED (in preparation)

22. R. Haag, Local Quantum Physics (Springer, Heidelberg, 1996)

23. K. Bardackci, B. Schroer, Local approximations in renormalizable and nonrenormalizable theories II. J. Math. Phys. 7, 16 (1966)

24. P. Leyland, J. Roberts, D. Testard, Duality for Quantum Free Fields (Centre de Physique Théorique, CNRS Marseille, 1978)

25. D. Buchholz, F. Ciolli, G. Ruzzi, E. Vasselli, The universal C*algebra of the electromagnetic field. arXiv: 1506.06603

26. B. Schroer, Fortschr. Phys. 173, 1527 (1963)

27. J. Fröhlich, G. Morchio, F. Strocchi, Phys. Lett. 89B, 61 (1979)

28. F. Bloch, A. Nordsieck, Phys. Rev. 52, 5 (1937)

29. D. Yenni, S. Frautschi, H. Suura, Ann. Phys. 13, 370(1961) 
30. D. Buchholz, J. Roberts, New light on infrared problems: sectors, statistics, symmetries and spectrum. arXiv:1304.2794

31. P.W. Higgs, Phys. Lett. 12, 132 (1964)

32. E.P. Wigner, On unitary representations of the inhomogeneous Lorentz group. Ann. Math. 40 (1939)
33. S. Weinberg, The Quantum Theory of Fields I (Cambridge University Press, Cambridge, 1991)

34. M. Dütsch, B. Schroer, J. Phys. A 33, 4317 (2000)

35. S. Hollands, Renormalized quantum Yang-Mills fields in curved spacetime. Rev. Math. Phys. 20, 1033 (2008). arXiv:0705.3340 